\title{
Synthesis, Structure and In Vitro Anti-Trypanosomal Activity of Non-Toxic Arylpyrrole-Based Chalcone Derivatives
}

\author{
Ayanda I. Zulu ${ }^{1}$, Ogunyemi O. Oderinlo ${ }^{1}{ }^{1}$, Cuan Kruger $^{1}$, Michelle Isaacs ${ }^{2}$, \\ Heinrich C. Hoppe ${ }^{2,3}{ }^{\mathbb{D}}$, Vincent J. Smith ${ }^{1,2}{ }^{\oplus}$, Clinton G. L. Veale ${ }^{4,5}(\mathbb{D}$ and \\ Setshaba D. Khanye $1,2,5, *$ (D) \\ 1 Department of Chemistry, Faculty of Science, Rhodes University, Grahamstown 6140, South Africa; \\ azulu50@gmail.com (A.I.Z.); oderinloyemi@yahoo.com (O.O.O.); g13k8200@campus.ru.ac.za (C.K.); \\ v.smith@ru.ac.za (V.J.S.) \\ 2 Centre for Chemico and Biomedicinal Research, Rhodes University, Grahamstown 6140, South Africa; \\ m.isaacs@ru.ac.za (M.I.); h.hoppe@ru.ac.za (H.C.H.) \\ 3 Department of Biochemistry and Microbiology, Faculty of Science, Rhodes University, Grahamstown 6140, \\ South Africa \\ 4 School of Chemistry and Physics, Pietermaritzburg Campus, University of KwaZulu-Natal, Private Bag X01, \\ Scottsville 3209, South Africa; VealeC@ukzn.ac.za \\ 5 Division of Pharmaceutical Chemistry, Faculty of Pharmacy, Rhodes University, Grahamstown 6140, \\ South Africa \\ * Correspondence: s.khanye@ru.ac.za; Tel.: +27-46-603-8397
}

Academic Editor: Andrew Tsotinis

Received: 21 March 2020; Accepted: 2 April 2020; Published: 4 April 2020

\begin{abstract}
With an intention of identifying chalcone derivatives exhibiting anti-protozoal activity, a cohort of relatively unexplored arylpyrrole-based chalcone derivatives were synthesized in moderate to good yields. The resultant compounds were evaluated in vitro for their potential activity against a cultured Trypanosoma brucei brucei 427 strain. Several compounds displayed mostly modest in vitro anti-trypanosomal activity with compounds $10 \mathrm{e}$ and $10 \mathrm{~h}$ emerging as active candidates with $\mathrm{IC}_{50}$ values of 4.09 and $5.11 \mu \mathrm{M}$, respectively. More importantly, a concomitant assessment of their activity against a human cervix adenocarcinoma (HeLa) cell line revealed that these compounds are non-toxic.
\end{abstract}

Keywords: arylpyrrole; chalcones; trypanosomiasis; Trypanosoma brucei; molecular hybridization

\section{Introduction}

Infectious diseases caused by kinetoplastid parasites are a serious health threat worldwide. Among these is human African trypanosomiasis (HAT), commonly referred to as sleeping sickness [1,2]. HAT has also been prioritized by the World Health Organization (WHO) in the list of neglected tropical diseases (NTDs) in 149 endemic countries [3-5]. A significantly large population mainly in poor rural locations of sub-Saharan Africa remains at risk of contracting the disease $[5,6]$. The causative agents of HAT are protozoan trypanosomes of the genus Trypanosoma: T. brucei rhodesiense and T. brucei gambiense $[7,8]$. These are transmitted by the insect vector tsetse fly of the Glossina species, which injects metacyclic trypomastigotes into the human host [9]. T. $b$. gambiense, which is common to west and central Africa, is responsible for $>98 \%$ of reported cases of HAT in the continent of Africa [10], whilst T. $b$. rhodesiense is found in eastern and southern Africa and accounts for less than $2 \%$ of reported cases.

Despite their discovery almost a century ago, the most successful agents in clinical use for the treatment of HAT are pentamidine, melarsoprol (Figure 1), suramin, and eflornithine [11]. However, these drugs suffer from some limitations, including poor oral bioavailability and complex and 
time-consuming intravenous (IV) administration, which is costly and requires highly skilled health practitioners and facilities that are limited in poverty-stricken rural areas [12,13], and thus impact the implementation of treatment [14]. Additionally, currently available anti-trypanosomal drugs have serious side effects, such as nephrotoxicity, hypertension, anemia, and neuropathy $[15,16]$. In the absence of effective vaccines to prevent infections inflicted by HAT, treatment efforts are solely reliant on chemotherapeutic intervention [3,17]. Treatment options are implemented such that for stage 1 of the disease pentamidine and suramin are utilized, despite their limited penetration of the central nervous system (CNS) [18]. For stage 2 of the disease, melarsoprol and eflornithine are the preferred treatment options, however, these drugs suffer from issues relating to toxicity and trypanosomal resistance [19]. Considering the urgent need to develop therapeutic alternatives that target both stages of trypanosomiasis, a partnership between Sanofi and Drug for Neglected Diseases initiative (DNDi) has led to the approval of fexinidazole (Figure 1) as an alternative therapeutic armament for tackling both stage 1 and 2 HAT [1,20]. However, the ever-growing concern regarding resistance against clinically approved drugs, and the potential for an epidemic outbreak, has resulted in several campaigns focused on identifying new compounds with novel modes of action to prevent and control trypanosomal infections [21-23].
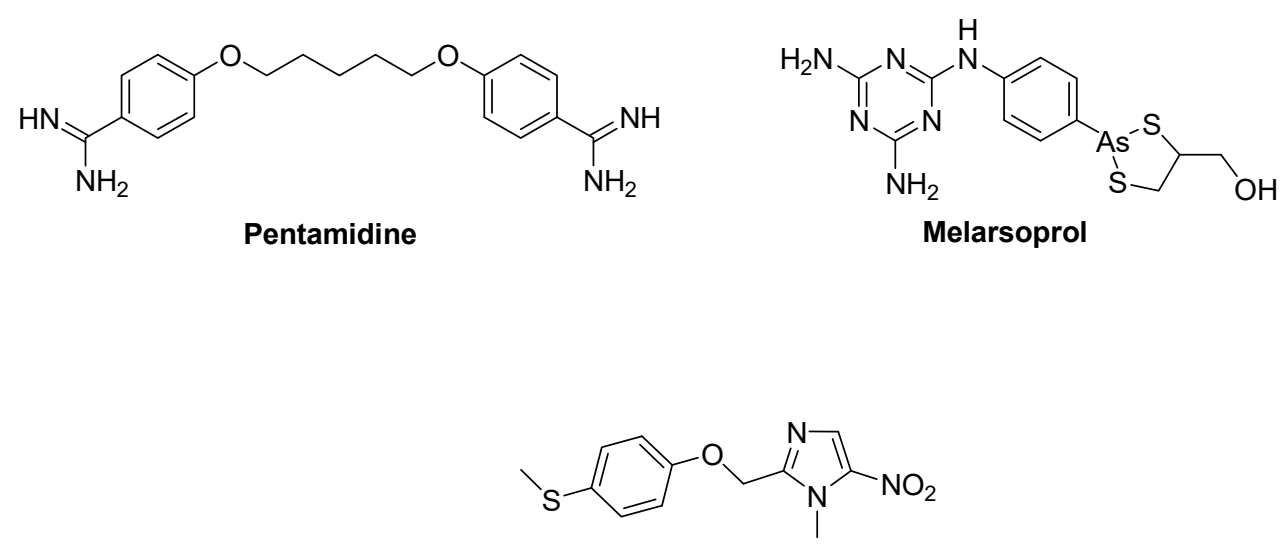

Fexinidazole

Figure 1. Examples of some drugs used for the treatment of human African sleeping sickness.

Chalcones are open-chain flavonoids, which feature a characteristic three carbon $\alpha, \beta$-unsaturated carbonyl system [24,25]. More importantly, chalcones are appealing as key synthetic intermediates and frameworks in the design of therapeutic tools for the treatment of various ailments including antimicrobial, antibacterial, antimalarial, anticancer, anti-inflammatory, anti-HIV, anti-Alzheimer's, etc. [24-32]. The structural modification of chalcones where the B-ring (Figure 2) is substituted with other bioactive fragments or units is a contemporary strategy that has been extensively utilized by various research groups involved in designing bioactive compounds for the treatment of different diseases [33]. A typical example is that of Zhou and co-workers, who prepared a series of novel chalcones by incorporating the benzoxaborole motif to form hybrid molecules (Figure 2) showing excellent in vivo activity in a murine model of T. $b$. brucei, with compound $\mathbf{1}$ emerging as the most active compound, with an $\mathrm{IC}_{50}$ value of $0.010 \mu \mathrm{g} / \mathrm{mL}$ [34].

In an effort to identify compounds with desirable potency against methicillin-resistant Staphylococcus aureus (MRSA), Osório and co-workers reported a focused library of chalcone derivatives, including compound 2, bearing an $\mathrm{N}$-arylpyrrole moiety [35]. Thus far, compound 2 remains unexplored for antiprotozoal activity, with no examples of analogues appearing in the literature. Importantly, in our context, several studies have shown that compounds containing the arylpyrrole framework display a broad spectrum of biological properties, including antiparasitic activities, as illustrated by the anti-malarial lead compound 3 [36]. Furthermore, numerous chalcone derivatives, including 
compounds 4 and 5, have been reported as possessing anti-bacterial and anti-tuberculosis activity [37]. In a separate study, Dave et al. explored chalcone derivatives in which the B-ring was substituted for a quinoline nucleus, for their potential as antiplasmodial agents. In that study, compound 6 displayed favorable anti-plasmodial potency, with the 5-bromo-2-hydroxy substitution pattern on ring A (portion in blue) appearing to be a crucial element of the pharmacophore [38].

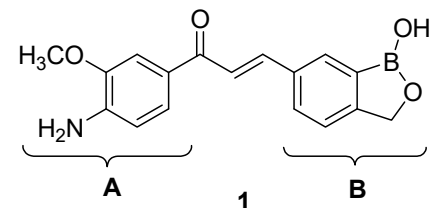<smiles>O=C(/C=C/c1ccccc1)c1cc(Br)ccc1O</smiles>

4<smiles>COc1ccc(OC)c(C(=O)/C=C/c2cc(C)n(-c3cccc(C(F)(F)F)c3)c2C)c1</smiles>

2<smiles>[R]c1ccc(N=C(/C=C/c2ccccc2)c2cc(Br)ccc2O)cc1</smiles>

5<smiles>[R]n1c(C)cc(/C=C/C(=O)c2cc(Br)ccc2O)c1C</smiles>

Target compounds

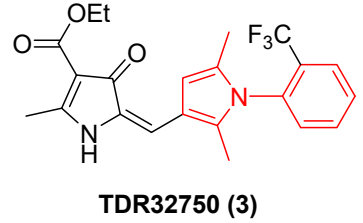<smiles>O=C(/C=C/c1cc2ccccc2nc1Cl)c1cc(Br)cc(Cl)c1O</smiles>

6

Figure 2. Examples of some bioactive chalcone derivatives, arylpyrroles, and target compounds.

Molecular hybridization, which combines two or more dissimilar pharmacophoric subunits to improve efficacy and other pharmaceutical profiles is becoming an attractive approach to design novel biologically active compounds [39-41]. Considering our ongoing research interests to identify and develop new antiparasitic compounds [42-44], we employed a molecular hybridization approach to design a target series of chalcone derivatives (Figure 2) featuring A ring with hydroxyl and bromine substituents at position 2 and 5, respectively, and arylpyrrole containing B ring system. Herein, we wish to report the synthesis of a representative series of new and non-toxic arylpyrrole-based chalcone derivatives, along with their in vitro anti-trypanosomal effects against the nagana T. $b$. brucei strain.

\section{Results and Discussion}

\subsection{Chemical Synthesis}

Reaction of commercially available 2,5-hexadione 7 with a variety of anilines under the standard Paal-Knorr condensation reaction conditions (Scheme 1) afforded starting arylpyrrole intermediates (see Supplementary Information) with yields varying between 38\%-81\% [45]. Subsequently, the Vilsmeier-Haack formylation of resultant arylpyrrole intermediates led to the formation of the desired aldehydes $\mathbf{8 a - k}$ in moderate to excellent yields [46]. Subjecting the commercially available 2,5-dimethyl- $1 \mathrm{H}$-pyrrole under similar Vilsmeier-Haack reaction conditions yielded compound $\mathbf{8 1}$ as a white solid in good yield. Treatment of prepared aldehydes 8a-1 with the 2-hydroxy-5-bromoacetophenone 9 under base-catalyzed Claisen-Schmidt condensation conditions, afforded the desired arylpyrrole-chalcone derivatives $\mathbf{1 0 a}-\mathbf{1}$, which were isolated in yields varying between $9 \%-71 \%$. Similarly, compound 11a was obtained in a poor yield by treating 8 with 2,5-dimethyl-1H-imidazole-4-carbaldehyde, while compound 11b was synthesized from the 2-chloro-6-methoxyquinoline-3-carbaldehyde at a 39\% yield, as previously described in the literature [38]. Further preliminary structure-activity relationship (SAR) investigations were conducted 
through structural modification of the ring A. The starting commercially available acetophenones were reacted with selected arylpyrrolecarbaldehydes $\mathbf{8 e}, \mathbf{8 h}$, and $\mathbf{8 i}$ under the same base-catalyzed Claisen-Schmidt reaction conditions (Scheme 1), to form the desired compounds 12-19 with yields in the range $5 \%-89 \%$.

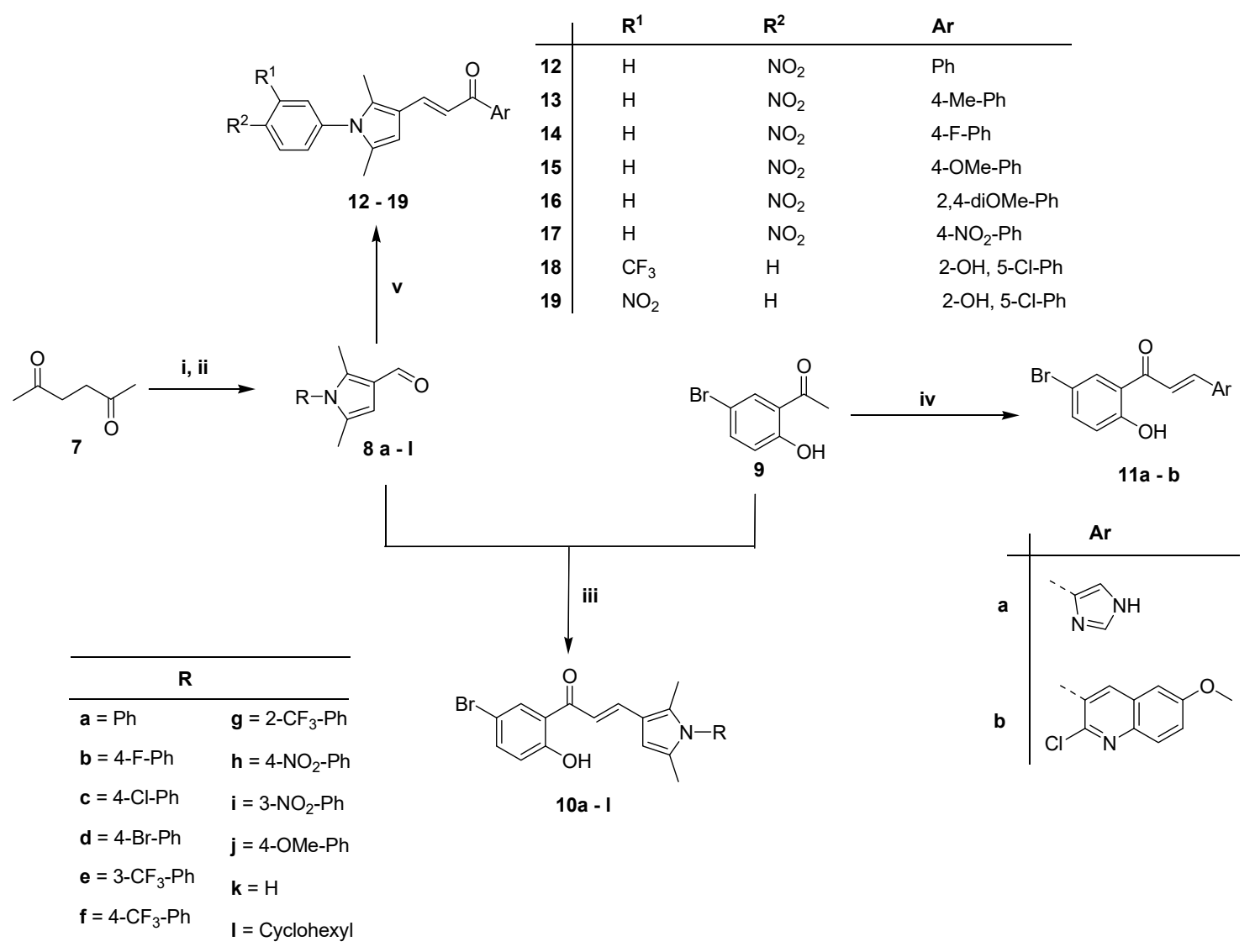

Scheme 1. Reagents and conditions: (i) Anilines or cyclohexalamine, solvent free, $160{ }^{\circ} \mathrm{C}, 16 \mathrm{~h}$; (ii) $\mathrm{POCl}_{3}, \mathrm{DMF}, 0^{\circ} \mathrm{C}-60{ }^{\circ} \mathrm{C}, 4 \mathrm{~h}$; (iii) $60 \% \mathrm{NaOH}, \mathrm{MeOH}, \mathrm{rt}, 12 \mathrm{~h}$; (iv) Aldehydes, $60 \% \mathrm{NaOH}, \mathrm{MeOH}$, $\mathrm{rt}, 12 \mathrm{~h}$.

All of the synthesized compounds were fully characterized using routine spectroscopic methods (NMR, IR and MS). The coupling constant $(J)$ of the $\alpha, \beta$-unsaturated protons featured values in the range $12-16 \mathrm{~Hz}$, thus indicating a trans configuration [47]. This observation was corroborated by the molecular structure of compound 10e, determined by single crystal X-ray diffraction analysis. Crystal and experimental data are summarized in Table S1 (Supplementary Information). Compound 10e crystallizes in the orthorhombic crystal system, in the centrosymmetric space group Pbca with eight molecules per unit cell, $Z=8$. The crystal structure of compound 10e (Figure 3 and Figure S1) confirmed unequivocally a trans configuration about the double bond $(C(8 \mathrm{~A})-\mathrm{C}(9 \mathrm{~A}))$. 


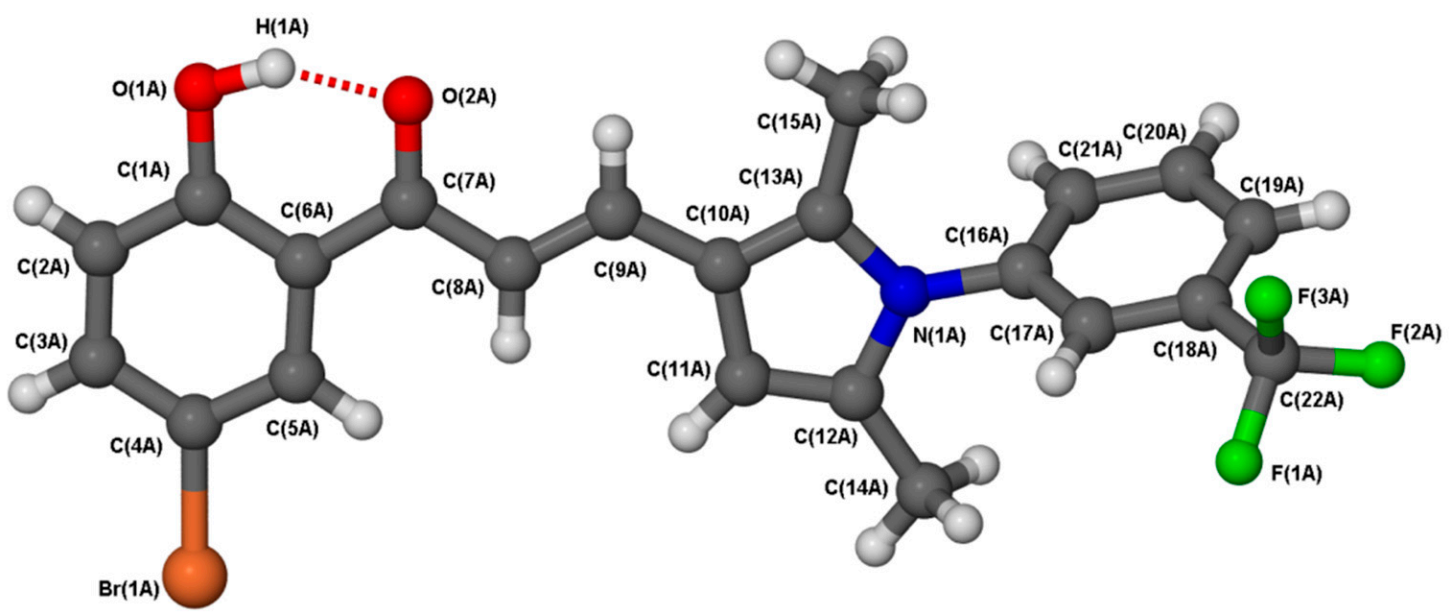

Figure 3. Molecular structure of 10e depicting the numbering scheme and the intramolecular hydrogen bond (shown here by the red dashed line). The hydrogen atoms on carbons were fixed in geometrically calculated while those bound to oxygen were freely refined. The structure was solved and refined to a final residual factor $R_{1}=0.0751$. All non-hydrogen atoms were refined anisotropically, with the ellipsoids drawn at the 50\% probability level (see Figure S1 in the Supplementary Materials).

From the structure of 10e, bond lengths and bond angles are comparable to chalcone derivatives, which have been reported in the literature [48]. The existence of an $\alpha, \beta$-unsaturated ketone is further confirmed by short $\mathrm{O}(2 \mathrm{~A})-\mathrm{C}(7 \mathrm{~A})$ and $\mathrm{C}(8 \mathrm{~A})-\mathrm{C}(9 \mathrm{~A})$ bond lengths of 1.244(7) $\AA$ and 1.359(6) $\AA$, respectively; and bond angles $\mathrm{O}(2 \mathrm{~A})-\mathrm{C}(7 \mathrm{~A})-\mathrm{C}(8 \mathrm{~A})$ and $\mathrm{C}(7 \mathrm{~A})-\mathrm{C}(8 \mathrm{~A})-\mathrm{C}(9 \mathrm{~A})$ of $121.2(5)^{\circ}$ and $120.8(4)^{\circ}$, respectively. More importantly, the structure exhibits intramolecular hydrogen bonding between the hydroxyl oxygen of ring A and the ketone adjacent to ring A. Introduction of intramolecular hydrogen bond (IMHB) into the bioactive compounds has been reported to have beneficial effects, including biological activity [49]. For example, Attram and co-workers synthesised a novel class of benzimidazoles containing an IMHB structural feature showing in vitro antiplasmodial activity against chloroquine-sensitive (NF54) and multi-drug resistant (K1) strains of the malaria parasite Plasmodium falciparum [50]. The aryl moiety on ring B of the chalcone is rotated about the $\mathrm{N}(1 \mathrm{~A})-\mathrm{C}(16 \mathrm{~A})$ bond with corresponding four torsion angles (Figure S2). The observed torsion angles suggest the deviation from planarity of the aryl group attached to the pyrrole framework. The crystal packing (Figure 4 ) of the molecule is stabilized by $\pi-\pi$ stacking interactions between phenyl rings (A) and between the phenyl and pyrrole rings $(\mathbf{B})$. The centroid-to-centroid distances $(\mathbf{A}, \mathbf{B})$ are approximately 3.927(3) $\AA$ and 3.731(2) $\AA$, which further confirms the intermolecular $\pi-\pi$ stacking interactions between chalcone molecules in the crystal structure [48]. Additionally, the supramolecular assembly is supported by weak $\mathrm{C}-\mathrm{H} \cdots \pi$ and $\mathrm{C}-\mathrm{F} \cdots \pi$ interactions between the trifluorophenyl ring moieties of the arylpyrrole (C) $[\mathrm{C}(21 \mathrm{~A})-\mathrm{H}(21 \mathrm{~A}) \cdots \pi$ is $2.95 \AA$ and $\mathrm{C}(22 \mathrm{~A})-\mathrm{F}(2 \mathrm{~A}) \cdots \pi$ is $3.32(1) \AA]$. 


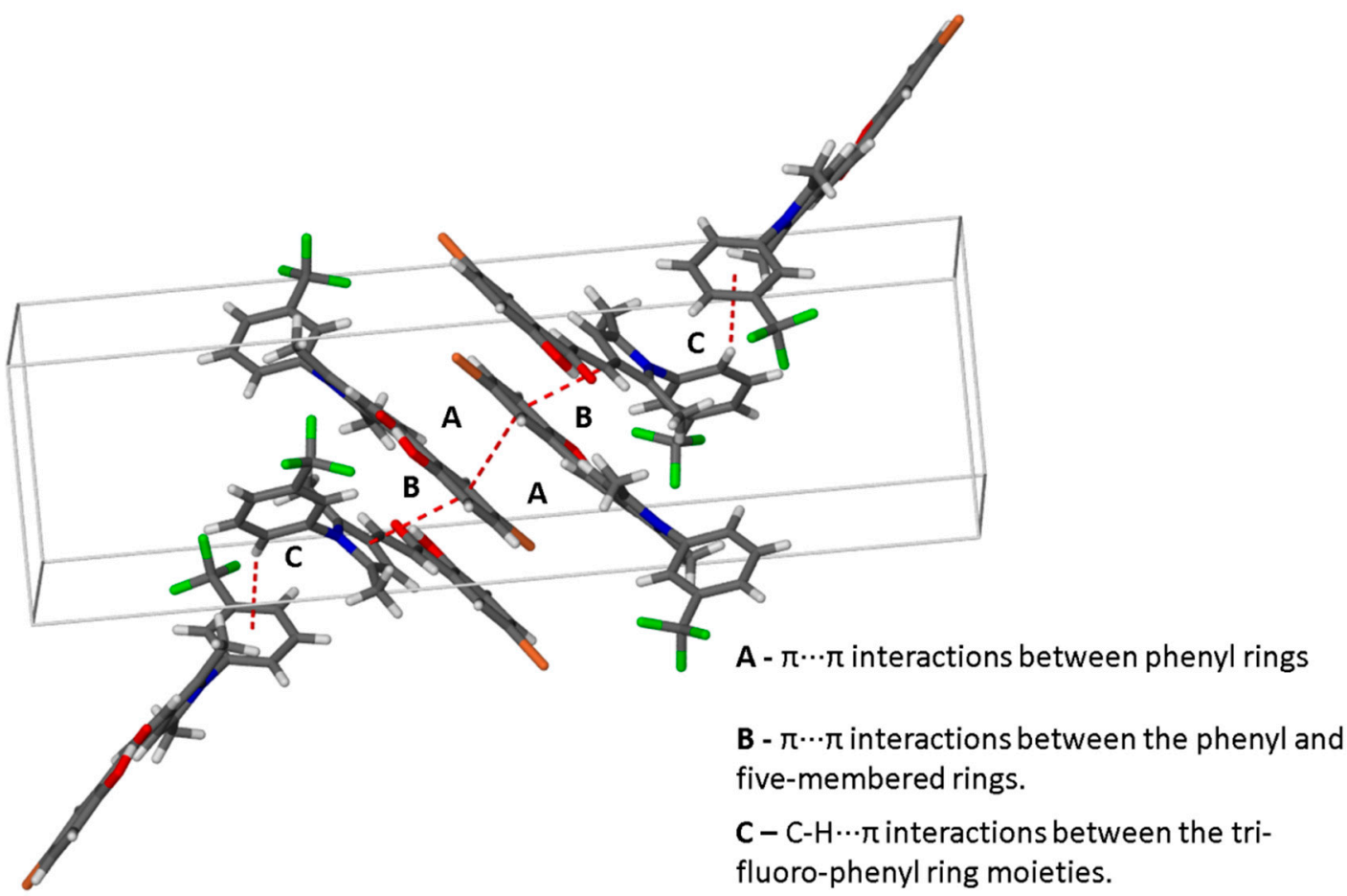

Figure 4. Packing diagram depicting $\pi \cdots \pi$ stacking and $\mathrm{C}-\mathrm{H} \cdots \pi$ interactions of $\mathbf{1 0 e}$ that run diagonally across the $b c$ plane. The C-F $\cdots \pi$ interactions have been omitted for clarity.

\subsection{In Vitro Biological Assessment}

A single concentration $(20 \mu \mathrm{M})$ cytotoxicity evaluation of all compounds was conducted in duplicates using a human cervix adenocarcinoma (HeLa) cell line, with emetine (EMT) included as a reference drug and cell viability relative to untreated cells assessed using resazurin. Compounds 10a-1 possessed negligible cytotoxicity effects against the HeLa cell line, and in general, $>80 \%$ HeLa cell viability was observed (see Table S2). The anti-trypanosomal activity of prepared compounds against nagana T. $b$. brucei (the subspecies responsible for African trypanosomiasis in cattle) parasite cultures were determined using the T. $b$. brucei 427 strain. Pentamidine (PMD), which is an existing drug for the treatment of trypanosomiasis, was included as a positive control drug. The trypanocidal activity of each compound was initially assessed at a fixed concentration of $20 \mu \mathrm{M}$, using resazurin as a read-out of cell viability after compound exposure. All of the compounds which inhibited cell viability below $25 \%$ relative to untreated controls at this concentration were taken forward for $\mathrm{IC}_{50}$ determination. From Table 1, the structural modification was initially performed on the arylpyrrole moiety (ring B) of the chalcones, while keeping the ring A unchanged. Compound 10a, with an unsubstituted aromatic ring attached to the arylpyrrole moiety, showed modest activity against $T . b$. brucei, with an $\mathrm{IC}_{50}$ value of $11.5 \mu \mathrm{M}$. Introduction of the fluorine, highly electronegative atom, at the para-position (10b) appeared to enhance the activity against $T . b$. brucei; however, the activity diminished when the fluorine was replaced by chlorine (10c). Despite 10d exhibiting similar activity to compound 10a, inclusion of the bromine substituent (10d) slightly restored activity comparable to 10c. The presence of the trifluoromethyl $\left(\mathrm{CF}_{3}\right)$ functionality at the para- position resulted in compound $\mathbf{1 0 e}$, whose activity closely mirrored $\mathbf{1 0 b}$. The positional isomer of $\mathbf{1 0 e}$ which features $\mathrm{CF}_{3}$ at the meta- position (10f) displayed reduced activity with an $\mathrm{IC}_{50}$ value of $11.4 \mu \mathrm{M}$, while the ortho substituted analogue $10 \mathrm{~g}$ showed moderate activity in line with compounds 10a, 10d, and 10f. Exchanging of $\mathrm{CF}_{3}$ with yet another electron withdrawing substituent, $\mathrm{NO}_{2}$ at the para position (10h), also showed improved biological activity with an $\mathrm{IC}_{50}<6 \mu \mathrm{M}$, while the meta isomer (10i) possessed modest activity, with an $\mathrm{IC}_{50}$ value slightly below $10 \mu \mathrm{M}$. Compound $\mathbf{1 0 j}$, which features an electron donating methoxy moiety, showed no activity. 
Table 1. In vitro bioassay data for chalcone derivatives $\mathbf{1 0 a}-\mathbf{1}, \mathbf{1 1 a}-\mathbf{b}$, and $\mathbf{1 2}-\mathbf{1 9}$ showing $\mathrm{IC}_{50}$ values for inhibition of T. b. brucei 427.

\begin{tabular}{|c|c|c|c|}
\hline \multirow{2}{*}{ Comp } & T. b. brucei & \multirow{2}{*}{ Comp } & \multirow{2}{*}{$\begin{array}{c}\text { T. b. brucei } \\
\mathrm{IC}_{50} / \mu \mathrm{M}\end{array}$} \\
\hline & $\mathrm{IC}_{50} / \mu \mathrm{M}$ & & \\
\hline $10 a$ & 11.5 & 101 & 11.5 \\
\hline $10 \mathrm{~b}$ & 6.9 & $11 a$ & na \\
\hline $10 \mathrm{c}$ & 40.0 & $11 b$ & 9.1 \\
\hline $10 d$ & 10.5 & 12 & na \\
\hline $10 \mathrm{e}$ & 4.1 & 13 & 44.3 \\
\hline $10 f$ & 11.4 & 14 & 36.5 \\
\hline $10 \mathrm{~g}$ & 10.0 & 15 & 12.3 \\
\hline $10 \mathrm{~h}$ & 5.1 & 16 & 10.6 \\
\hline $10 \mathrm{i}$ & 9.3 & 17 & na \\
\hline $10 \mathrm{j}$ & na & 18 & 9.3 \\
\hline $10 \mathrm{k}$ & na & 19 & 11.7 \\
\hline PMD & $0.68 \mathrm{nM}$ & - & - \\
\hline
\end{tabular}

PMD = Pentamidine. na $=$ not active (compounds producing a cell viability of $\geq 50 \%$ in the preliminary single point assay at $20 \mu \mathrm{M})$.

Compound 10k, which lacked an aromatic ring substituent on the pyrrole ring, showed a complete loss of activity against the T. b. brucei strain, while compound 101, with a saturated cyclohexyl ring instead of either a substituted or non-substituted aromatic ring, showed no improvement in terms of potency, and its $\mathrm{IC}_{50}$ value was comparable to that of compounds 10a and 10f. Compounds 11a and 11b, which featured aromatic replacements of the arylpyrrole moiety, were evaluated for trypanocidal activity. While 11a showed no activity, compound $\mathbf{1 1 b}$, with a quinoline heterocyclic unit, was found to be moderately active against trypanosomes, with an $\mathrm{IC}_{50}$ value of $9.1 \mu \mathrm{M}$. Having identified compounds 10e and 10h as the most active compounds in our initial cohort, we proceeded to explore compounds which maintained the arylpyrrole substituents of either compounds $10 \mathrm{e}$ and $\mathbf{1 0 h}$, whilst replacing the 2-hydro-5-bromo-substituted aromatic moiety. These compounds (12-19) showed moderate inhibitory activity against $T . b$. brucei, with $\mathrm{IC}_{50}$ values ranging between $9.3-44.3 \mu \mathrm{M}$ (Table 1). Despite moderate activity, the data suggest that a combination of the hydroxyl group at position 2 and the bromine at position 5 in the ring A is necessary for activity against the T. b. brucei parasite.

\section{Materials and Methods}

\subsection{General Information}

All commercially available reagents were purchased from chemical suppliers, Sigma-Adrich (Pty) Ltd. (Johannesburg, South Africa) and Merck (Pty) Ltd. (Johannesburg, South Africa), and were used without further purification unless stated otherwise. Solvents were distilled before use. The progress of reactions were monitored by thin layer chromatography (TLC) using Merck $\mathrm{F}_{254}$ silica gel plates (Merck, Johannesburg, South Africa) supported on aluminium, and were visualized under ultra-violet (UV 254 and $366 \mathrm{~nm}$ ) light, and, where necessary, stained in an iodine tank. The crude compounds were purified by a silica gel column chromatography using Merck Kieselgel $60 \AA$ A: 70-230 $(0.068-0.2 \mathrm{~mm})$ silica gel mesh. NMR $\left({ }^{1} \mathrm{H}\right.$ and $\left.{ }^{13} \mathrm{C}\right)$ spectra were recorded on Bruker Biospin 300, 400, or $600 \mathrm{MHz}$ spectrometer. Chemical shifts are reported in ppm and are referenced internally using residual solvent signals (DMSO- $d_{6} \delta_{\mathrm{H}} 2.50, \delta_{\mathrm{C}} 39.5 \mathrm{ppm} ; \mathrm{CDCl}_{3} \delta_{\mathrm{H}} 7.26, \delta_{\mathrm{C}} 77.0 \mathrm{ppm}$ ). The coupling constants are given in Hertz. High-resolution mass spectrometry (HRMS) was performed on a Waters API Q-TOF Ultima spectrometer (Stellenbosch University, Stellenbosch, South Africa). The IR spectra were recorded on PerkinElmer Spectrum 100 FT-IR Spectrometer (Johannesburg, South Africa) in the mid-IR range $\left(640-4000 \mathrm{~cm}^{-1}\right)$. Melting points were measured using a Reichert hot-stage apparatus and are uncorrected. Elemental microanalysis was performed on Elementar Analysensysteme varioMICRO V1.6.2 GmbH analysis system. The calculated values were determined using an online Elemental 
Composition Calculator from Microanalysis Laboratory, School of Chemical Sciences, University of Illinois at Urbana, USA [51]. Herein, compound 11b was synthesized from the starting ketone 8 and 2-chloro-6-methoxyquinoline-3-carbaldehyde, according to the method previously described in the literature [38].

\subsection{General Procedure for the Synthesis of Arylpyrrole-Chalcone Hybrids}

A $60 \% \mathrm{NaOH}(10 \mathrm{~mL})$ solution was cooled in an ice bath while stirring, and 5-bromo2-hydroxyacetophenone (1 eq.) was slowly added and the reaction mixture allowed stirring for $20 \mathrm{~min}$. Thereafter, an appropriate aldehyde (1 eq.) dissolved in $\mathrm{MeOH}(10 \mathrm{~mL})$ was added, and the resultant reaction mixture allowed to stir for $12 \mathrm{~h}$ at room temperature. The reaction progress was monitored by TLC (Hex: EtOAc), which showed the formation of a new spot, while the traces of starting material were still visible. After the completion of the reaction, the product precipitated out of the solution, which was filtered and washed with ice cold water and methanol. In cases where there was no precipitate formed, the product was poured onto water and extracted with ethyl acetate. The organic layer was dried $\left(\mathrm{Na}_{2} \mathrm{SO}_{4}\right)$, filtered, and evaporated the solvent in vacuo. The resulting crude product was purified by a silica gel column chromatography to give products as solids.

(E)-1-(5-Bromo-2-hydroxyphenyl)-3-(2,5-dimethyl-1-phenyl-1H-pyrrol-3-yl)prop-2-en-1-one 10a, Yellow crystalline solid. Yield $=24 \%$. m.p. $148{ }^{\circ} \mathrm{C}-150{ }^{\circ} \mathrm{C} .{ }^{1} \mathrm{H}$ NMR $\left(300 \mathrm{MHz}, \mathrm{CDCl}_{3}\right): \delta_{\mathrm{H}} 13.3(1 \mathrm{H}$, s, OH), $8.07\left(1 \mathrm{H}, \mathrm{d}, J=14.7 \mathrm{~Hz}, \mathrm{H}_{\alpha \beta}\right), 8.00(1 \mathrm{H}, \mathrm{d}, J=2.1 \mathrm{~Hz}, \mathrm{ArH}), 7.53-7.47$ (4H, m, ArHs), 7.23-7.20 (2H, m, ArHs), $7.16\left(1 \mathrm{H}, \mathrm{d}, J=14.7 \mathrm{~Hz}, \mathrm{H}_{\alpha \beta}\right), 6.90(1 \mathrm{H}, \mathrm{d}, J=9.0 \mathrm{~Hz}, \mathrm{ArH}), 6.41(1 \mathrm{H}, \mathrm{s}$, pyrrole-H), $2.18\left(3 \mathrm{H}, \mathrm{s}, \mathrm{CH}_{3}\right), 2.04\left(3 \mathrm{H}, \mathrm{s}, \mathrm{CH}_{3}\right) .{ }^{13} \mathrm{C} \mathrm{NMR}\left(75 \mathrm{MHz} \mathrm{CDCl}_{3}\right): \delta_{\mathrm{C}} 192.6,162.50,140.7,138.0,137.7$, 136.9, 132.1, 131.7, 129.7, 128.9, 128.1, 121.9, 120.5, 118.0, 112.9, 110.2, 104.4,12.9, 11.2. HRMS (ESI $\left.{ }^{+}\right) \mathrm{m} / \mathrm{z}$ (ESI-HRMS) found 396.0597, calcd for $\mathrm{C}_{21} \mathrm{H}_{19} \mathrm{NO}_{2} \mathrm{Br}: 396.0594[\mathrm{M}+\mathrm{H}]^{+}$. Anal. Found: $\mathrm{C}, 62.79 ; \mathrm{H}$, 4.13; N, 3.09\%. Anal. calcd. $\mathrm{C}_{21} \mathrm{H}_{18} \mathrm{NO}_{2} \mathrm{Br} \cdot 0.5 \mathrm{H}_{2} \mathrm{O}$ : C, 62.23; $\mathrm{H}, 4.73 ; \mathrm{N}, 3.46 \%$.

(E)-1-(5-Bromo-2-hydroxyphenyl)-3-(1-(4-fluorophenyl)-2,5-dimethyl-1H-pyrrol-3-yl)prop-2-en-1-one 10b, Yellow solid. Yield $=39 \%$. m.p. $135{ }^{\circ} \mathrm{C}-138{ }^{\circ} \mathrm{C} .{ }^{1} \mathrm{H}$ NMR $\left(400 \mathrm{MHz}, \mathrm{CDCl}_{3}\right): \delta_{\mathrm{H}} 13.3(1 \mathrm{H}, \mathrm{s}$, $\mathrm{OH}), 8.05\left(1 \mathrm{H}, \mathrm{d}, J=14.8 \mathrm{~Hz}, \mathrm{H}_{\alpha \beta}\right), 7.99(1 \mathrm{H}, \mathrm{d}, J=2.4 \mathrm{~Hz}, \mathrm{ArH}), 7.51(1 \mathrm{H}, \mathrm{dd}, J=2.4$ and $8.8 \mathrm{~Hz}$, ArH), 7.34-7.26 (4H, m, ArHs), $7.16\left(1 \mathrm{H}, \mathrm{d}, J=14.8 \mathrm{~Hz}, \mathrm{H}_{\alpha \beta}\right), 6.90(1 \mathrm{H}, \mathrm{d}, J=9.2 \mathrm{~Hz}, \mathrm{ArH}), 6.40(1 \mathrm{H}$, s, pyrrole-H), $2.17\left(3 \mathrm{H}, \mathrm{s}, \mathrm{CH}_{3}\right), 2.03\left(3 \mathrm{H}, \mathrm{s}, \mathrm{CH}_{3}\right) .{ }^{13} \mathrm{C} \mathrm{NMR}\left(100 \mathrm{MHz} \mathrm{CDCl}_{3}\right): \delta_{\mathrm{C}} 192.5,163.8(2 \mathrm{C})$, 161.2 (2C), 140.5, 138.1, 136.7, 133.6, 132.0, 131.6, 129.8, 121.8, 120.5, 118.1, 116.7, 113.1, 110.3, 104.5, 12.9, 11.2. $\mathrm{m} / \mathrm{z}$ (ESI-HRMS) found 414.0505 , calcd for $\mathrm{C}_{21} \mathrm{H}_{18} \mathrm{BrFNO}_{2}: 414.0499[\mathrm{M}+\mathrm{H}]^{+}$. Anal. Found: C, 56.92; $\mathrm{H}, 2.74 ; \mathrm{N}, 2.87 \%$. Anal. calcd. $\mathrm{C}_{21} \mathrm{H}_{17} \mathrm{NO}_{2} \mathrm{BrF} \cdot 2 \mathrm{H}_{2} \mathrm{O}: \mathrm{C}, 56.01 ; \mathrm{H}, 4.70 ; \mathrm{N}, 3.11 \%$.

(E)-1-(5-Bromo-2-hydroxyphenyl)-3-(1-(4-chlorophenyl)-2,5-dimethyl-1H-pyrrol-3-yl)prop-2-en-1-one 10c, Yellow crystalline solid. Yield $=53 \%$. m.p. $127{ }^{\circ} \mathrm{C}-129{ }^{\circ} \mathrm{C} .{ }^{1} \mathrm{H} \mathrm{NMR}\left(300 \mathrm{MHz}, \mathrm{CDCl}_{3}\right): \delta_{\mathrm{H}}$ $13.3(1 \mathrm{H}, \mathrm{s}, \mathrm{OH}), 8.03\left(1 \mathrm{H}, \mathrm{d}, J=14.4 \mathrm{~Hz}, \mathrm{H}_{\alpha \beta}\right), 8.00(1 \mathrm{H}, \mathrm{d}, J=2.4 \mathrm{~Hz}, \mathrm{ArH}), 7.53-7.48\left(3 \mathrm{H}, \mathrm{m}, \mathrm{H}_{\alpha \beta}\right.$, ArHs), 7.19-7.14 (3H, m, ArHs), $6.90(1 \mathrm{H}, \mathrm{d}, J=9.0 \mathrm{~Hz}, \mathrm{ArH}), 6.40\left(1 \mathrm{H}, \mathrm{s}\right.$, pyrrole-H), $2.17\left(3 \mathrm{H}_{1}, \mathrm{c}, \mathrm{CH}_{3}\right)$, $2.03\left(3 \mathrm{H}, \mathrm{s}, \mathrm{CH}_{3}\right) .{ }^{13} \mathrm{C} \mathrm{NMR}\left(75 \mathrm{MHz} \mathrm{CDCl}_{3}\right): \delta_{\mathrm{C}} 192.6,162.5,140.4,138.1,136.5,136.2,134.9,131.9$, 131.7, 130.0, 129.4, 121.9, 120.6, 118.3, 113.4, 110.3, 104.7, 12.9, 11.3. HRMS (ESI $\left.{ }^{+}\right) \mathrm{m} / z$ (ESI-HRMS) found 430.0200, calcd for $\mathrm{C}_{21} \mathrm{H}_{18} \mathrm{NO}_{2} \mathrm{ClBr}$ : $430.0204[\mathrm{M}+\mathrm{H}]^{+}$. Anal. Found: C, 58.15; H, 3.71; N, 3.19\%. Anal. calcd. $\mathrm{C}_{21} \mathrm{H}_{17} \mathrm{NO}_{2} \mathrm{BrCl}$ : C, 58.56; $\mathrm{H}, 3.98 ; \mathrm{N}, 3.25 \%$.

(E)-1-(5-Bromo-2-hydroxyphenyl)-3-(1-(4-bromophenyl)-2,5-dimethyl-1H-pyrrol-3-yl)prop-2-en-1-one 10d, Yellow crystalline solid. Yield $=45 \%$. m.p. $141{ }^{\circ} \mathrm{C}-144{ }^{\circ} \mathrm{C} .{ }^{1} \mathrm{H}$ NMR $\left(300 \mathrm{MHz}, \mathrm{CDCl}_{3}\right): \delta_{\mathrm{H}} 13.3(1 \mathrm{H}, \mathrm{s}$, $\mathrm{OH}), 8.04\left(1 \mathrm{H}, \mathrm{d}, J=15.0 \mathrm{~Hz}, \mathrm{H}_{\alpha \beta}\right), 7.99(1 \mathrm{H}, \mathrm{d}, J=2.4 \mathrm{~Hz}, \mathrm{ArH}), 7.65(2 \mathrm{H}, \mathrm{d}, J=8.7 \mathrm{~Hz}, \mathrm{ArHs}), 7.52$ $(1 \mathrm{H}, \mathrm{dd}, J=2.4$ and $9.0 \mathrm{~Hz}, \mathrm{ArH}), 7.16\left(1 \mathrm{H}, \mathrm{d}, J=14.7 \mathrm{~Hz}, \mathrm{H}_{\alpha \beta}\right), 7.10(2 \mathrm{H}, \mathrm{d}, J=8.7 \mathrm{~Hz}, \mathrm{ArHs}), 6.90$ $(1 \mathrm{H}, \mathrm{d}, J=8.7 \mathrm{~Hz}, \mathrm{ArH}), 6.40\left(1 \mathrm{H}, \mathrm{s}\right.$, pyrrole-H), $2.18\left(3 \mathrm{H}, \mathrm{s}, \mathrm{CH}_{3}\right), 2.03\left(3 \mathrm{H}, \mathrm{s}, \mathrm{CH}_{3}\right) .{ }^{13} \mathrm{C} \mathrm{NMR}(75 \mathrm{MHz}$, $\left.\mathrm{CDCl}_{3}\right): \delta_{\mathrm{C}} 192.4,162.4,140.2,138.0,136.6,136.2,132.8,131.6,131.5,129.6,122.7,121.7,120.4,118.1$, 113.2, 110.1, 104.6, 12.3, 11.0. HRMS (ESI ${ }^{+}$) $m / z$ (ESI-HRMS) found 473.9701, calcd for $\mathrm{C}_{21} \mathrm{H}_{18} \mathrm{NO}_{2} \mathrm{Br}_{2}$ : 
473.9699, $[\mathrm{M}+\mathrm{H}]^{+}$. Anal. Found: $\mathrm{C}, 52.65 ; \mathrm{H}, 3.19 ; \mathrm{N}, 2.89 \%$. Anal. calcd. $\mathrm{C}_{21} \mathrm{H}_{17} \mathrm{NO}_{2} \mathrm{Br}_{2} \cdot 0.5 \mathrm{H}_{2} \mathrm{O}: \mathrm{C}$, $52.08 ; \mathrm{H}, 3.75 ; \mathrm{N}, 2.89 \%$.

(E)-1-(5-Bromo-2-hydroxyphenyl)-3-(2,5-dimethyl-1-(3-(trifluoromethyl)phenyl)-1H-pyrrol-3-yl)prop-2-en-1-one 10e, Yellow crystalline solid. Yield $=20 \%$. m.p. $135^{\circ} \mathrm{C}-137^{\circ} \mathrm{C} .{ }^{1} \mathrm{H}$ NMR $\left(300 \mathrm{MHz}, \mathrm{CDCl}_{3}\right): \delta_{\mathrm{H}} 13.2$ $(1 \mathrm{H}, \mathrm{s}, \mathrm{OH}), 8.04\left(1 \mathrm{H}, \mathrm{d}, J=15.0 \mathrm{~Hz}, \mathrm{H}_{\alpha \beta}\right), 8.00(1 \mathrm{H}, \mathrm{d}, J=2.4 \mathrm{~Hz}, \mathrm{ArH}), 7.77-7.75(1 \mathrm{H}, \mathrm{m}, \mathrm{ArH}), 7.67$ $(1 \mathrm{H}, \mathrm{d}, J=7.8 \mathrm{~Hz}, \mathrm{Ar}), 7.54-7.50(2 \mathrm{H}, \mathrm{m} \mathrm{ArHs}), 7.44(1 \mathrm{H}, \mathrm{d}, J=7.8 \mathrm{~Hz}, \mathrm{ArH}), 7.18(1 \mathrm{H}, \mathrm{d}, J=14.7 \mathrm{~Hz}$, $\left.\mathrm{H}_{\alpha \beta}\right), 6.91(1 \mathrm{H}, \mathrm{d}, J=9.0 \mathrm{~Hz}, \mathrm{ArH}), 6.43(1 \mathrm{H}, \mathrm{s}$, pyrrole- $\mathrm{H}), 2.19\left(3 \mathrm{H}, \mathrm{s}, \mathrm{CH}_{3}\right), 2.05\left(3 \mathrm{H}, \mathrm{s}, \mathrm{CH}_{3}\right) ;{ }^{13} \mathrm{C}$ NMR $\left(75 \mathrm{MHz}, \mathrm{CDCl}_{3}\right): \delta_{\mathrm{C}} 192.4,162.2,140.2,138.2,138.0,136.0,131.6,131.6,131.4,130.3,125.5$, 125.2, 124.9, 121.6, 120.4, 118.4, 114.9, 113.6, 110.1, 105.0, 12.8, 11.1. $\mathrm{m} / \mathrm{z}$ (ESI-HRMS) found 464.0456 calcd for $\mathrm{C}_{22} \mathrm{H}_{16} \mathrm{NO}_{2} \mathrm{~F}_{3} \mathrm{Br}$ : $464.0468[\mathrm{M}+\mathrm{H}]^{+}$. Anal. Found: $\mathrm{C}, 57.24 ; \mathrm{H}, 4.76 ; \mathrm{N}, 2.86 \%$. Anal. calcd. $\mathrm{C}_{22} \mathrm{H}_{17} \mathrm{NO}_{2} \mathrm{BrF}_{3} \cdot 0.5 \mathrm{CH}_{3} \mathrm{OH}: \mathrm{C}, 56.27 ; \mathrm{H}, 3.99 . ; \mathrm{N}, 2.92 \%$.

(E)-1-(5-Bromo-2-hydroxyphenyl)-3-(2,5-dimethyl-1-(4-(trifluoromethyl)phenyl)-1H-pyrrol-3-yl)prop-2-en-1-one 10f, Yellow crystalline solid. Yield $=70 \%$. m.p. $157^{\circ} \mathrm{C}-159^{\circ} \mathrm{C} .{ }^{1} \mathrm{H} \mathrm{NMR}\left(400 \mathrm{MHz}, \mathrm{CDCl}_{3}\right): \delta_{\mathrm{H}} 13.2$ $(1 \mathrm{H}, \mathrm{s}, \mathrm{OH}), 8.04\left(1 \mathrm{H}, \mathrm{d}, J=14.8 \mathrm{~Hz}, \mathrm{H}_{\alpha \beta}\right), 8.00(1 \mathrm{H}, \mathrm{d}, J=2.4 \mathrm{~Hz}, \mathrm{ArH}), 7.80(2 \mathrm{H}, \mathrm{d}, J=8.4 \mathrm{~Hz}$, ArHs), $7.52(1 \mathrm{H}, \mathrm{dd}, J=2.4$ and $8.8 \mathrm{~Hz}, \mathrm{ArH}), 7.37(2 \mathrm{H}, \mathrm{d}, J=8.4 \mathrm{~Hz}, \mathrm{ArHs}), 7.18(1 \mathrm{H}, \mathrm{d}, J=14.8 \mathrm{~Hz}$, $\left.\mathrm{H}_{\alpha \beta}\right), 6.91(1 \mathrm{H}, \mathrm{d}, J=8.8 \mathrm{~Hz}, \mathrm{ArH}), 6.43\left(1 \mathrm{H}, \mathrm{s}\right.$, pyrrole-H), $2.19\left(3 \mathrm{H}, \mathrm{s}, \mathrm{CH}_{3}\right), 2.05\left(3 \mathrm{H}, \mathrm{s}, \mathrm{CH}_{3}\right)$. ${ }^{13} \mathrm{C}$ NMR $\left(100 \mathrm{MHz}, \mathrm{CDCl}_{3}\right): \delta_{\mathrm{C}} 192.6,162.5,140.1,138.2,136.1,131.7,131.3,130.9,128.8,126.9$, 125.1, 122.4, 121.81, 120.6, 118.6, 113.8, 110.3, 105.2, 12.9, 11.2. $\mathrm{m} / \mathrm{z}$ (ESI-HRMS) found 464.0468, calcd for $\mathrm{C}_{22} \mathrm{H}_{18} \mathrm{NO}_{2} \mathrm{BrF}_{3}: 464.0468[\mathrm{M}+\mathrm{H}]^{+}$. Anal. Found: $\mathrm{C}, 56.60 ; \mathrm{H}, 3.88 ; \mathrm{N}, 2.96 \%$. Anal. calcd. $\mathrm{C}_{22} \mathrm{H}_{17} \mathrm{NO}_{2} \mathrm{BrF}_{3} \cdot 0.5 \mathrm{CH}_{3} \mathrm{OH}: \mathrm{C}, 56.27 ; \mathrm{H}, 3.99 ; \mathrm{N}, 2.92 \%$.

(E)-1-(5-Bromo-2-hydroxyphenyl)-3-(2,5-dimethyl-1-(2-(trifluoromethyl)phenyl)-1H-pyrrol-3-yl)prop-2-en-1-one 10g, Yellow crystalline solid. Yield $=71 \%$. m.p. $115^{\circ} \mathrm{C}-118^{\circ} \mathrm{C} .{ }^{1} \mathrm{H} \mathrm{NMR}\left(400 \mathrm{MHz}, \mathrm{CDCl}_{3}\right): \delta_{\mathrm{H}} 13.3$ $\left(1 \mathrm{H}, \mathrm{s}, \mathrm{H}_{13}\right), 8.04\left(1 \mathrm{H}, \mathrm{d}, J=14.7 \mathrm{~Hz}, \mathrm{H}_{\alpha \beta}\right), 8.00(1 \mathrm{H}, \mathrm{d}, J=2.4 \mathrm{~Hz}, \mathrm{ArH}), 7.89-7.86(1 \mathrm{H}, \mathrm{m}, \mathrm{ArH})$, 7.76-7.71 (1H, m, ArH), 7.68-7.63 (1H, m, ArH), 7.51 (1H, dd, J = 2.4 and 9.0 Hz, ArH), 7.32-7.27 (1H, $\mathrm{m}, \mathrm{ArH}), 7.17\left(1 \mathrm{H}, \mathrm{d}, J=14.7 \mathrm{~Hz}, \mathrm{H}_{\alpha \beta}\right), 6.90(1 \mathrm{H}, \mathrm{d}, J=8.7 \mathrm{~Hz}, \mathrm{ArH}), 6.41(1 \mathrm{H}, \mathrm{s}$, pyrrole-H), 2.09 $\left(3 \mathrm{H}, \mathrm{s}, \mathrm{CH}_{3}\right), 1.95\left(3 \mathrm{H}, \mathrm{s}, \mathrm{CH}_{3}\right) .{ }^{13} \mathrm{C}$ NMR $\left(75 \mathrm{MHz} \mathrm{CDCl}_{3}\right): \delta_{\mathrm{C}} 192.7,162.5,140.5,138.1,137.8,135.8$, 133.4, 132.9, 131.7, 131.3, 129.9, 129.0, 127.8, 121.8, 120.5, 118.3, 113.4, 110.4, 110.3, 104.4, 12.5, 10.9. m/z (ESI-HRMS) found 462.0299, calcd for $\mathrm{C}_{22} \mathrm{H}_{16} \mathrm{NO}_{2} \mathrm{~F}_{3} \mathrm{Br}$ : 462.0322 [M-H] ${ }^{+}$. Anal. Found: C, 57.20; $\mathrm{H}$, 1.94; N, 2.95\%. Anal. calcd. $\mathrm{C}_{22} \mathrm{H}_{17} \mathrm{NO}_{2} \mathrm{BrF}_{3}$ : C, 56.91; $\mathrm{H}, 3.69 ; \mathrm{N}, 3.02 \%$.

(E)-1-(5-Bromo-2-hydroxyphenyl)-3-(2,5-dimethyl-1-(4-nitrophenyl)-1H-pyrrol-3-yl)prop-2-en-1-one 10h, Yellow crystalline solid. Yield $=37 \%$. m.p. $168{ }^{\circ} \mathrm{C}-170{ }^{\circ} \mathrm{C} .{ }^{1} \mathrm{H}$ NMR $\left(300 \mathrm{MHz}, \mathrm{CDCl}_{3}\right): \delta_{\mathrm{H}}$ $13.2(1 \mathrm{H}, \mathrm{s}, \mathrm{OH}), 8.41(2 \mathrm{H}, \mathrm{d}, J=8.8 \mathrm{~Hz}, \mathrm{ArHs}), 8.04-7.99\left(2 \mathrm{H}, \mathrm{m}, \mathrm{ArH}, \mathrm{H}_{\alpha \beta}\right), 7.53(1 \mathrm{H}, \mathrm{dd}, J=2.4$ and $8.8 \mathrm{~Hz}, \mathrm{ArH}), 7.43(2 \mathrm{H}, \mathrm{d}, J=8.8 \mathrm{~Hz}, \mathrm{ArHs}), 7.19\left(1 \mathrm{H}, \mathrm{d}, J=14.8 \mathrm{~Hz}, \mathrm{H}_{\alpha \beta}\right), 6.91(1 \mathrm{H}, \mathrm{d}, J=8.8 \mathrm{~Hz}$, ArH), $6.45\left(1 \mathrm{H}, \mathrm{s}\right.$, pyrrole-H), $2.21\left(3 \mathrm{H}, \mathrm{s}, \mathrm{CH}_{3}\right), 2.10\left(3 \mathrm{H}, \mathrm{s}, \mathrm{CH}_{3}\right) .{ }^{13} \mathrm{C} \mathrm{NMR}\left(75 \mathrm{MHz}, \mathrm{CDCl}_{3}\right): \delta_{\mathrm{C}} 192.6$, 162.5, 147.6, 143.3, 139.6, 138.3, 135.6, 131.7, 131.4, 129.1, 125.2, 121.7, 120.6, 119.0, 114.2, 110.3, 105.7, 13.0, 11.3. $\mathrm{m} / \mathrm{z}$ found 441.0444 , calcd for $\mathrm{C}_{21} \mathrm{H}_{18} \mathrm{~N}_{2} \mathrm{O}_{4} \mathrm{Br}: 441.0444[\mathrm{M}+\mathrm{H}]^{+}$.

(E)-1-(5-bromo-2-hydroxyphenyl)-3-(2,5-dimethyl-1-(3-nitrophenyl)-1H-pyrrol-3-yl)prop-2-en-1-one 10i, Yellow crystalline solid. Yield $=68 \%$. m.p. $102{ }^{\circ} \mathrm{C}-104{ }^{\circ} \mathrm{C} .{ }^{1} \mathrm{H}$ NMR $\left(300 \mathrm{MHz}, \mathrm{CDCl}_{3}\right): \delta_{\mathrm{H}} 13.2(1 \mathrm{H}$, $\mathrm{s}, \mathrm{OH}), 8.39-8.35(1 \mathrm{H}, \mathrm{m}, \mathrm{ArH}), 8.14(1 \mathrm{H}, \mathrm{t}, J=2.1 \mathrm{~Hz}, \mathrm{ArH}), 8.06-8.00\left(2 \mathrm{H}, \mathrm{m}, \mathrm{ArH}, \mathrm{H}_{\alpha \beta}\right), 7.75$ $(1 \mathrm{H}, \mathrm{t}, J=7.8 \mathrm{~Hz}, \mathrm{Ar}), 7.62-7.58(1 \mathrm{H}, \mathrm{m}, \mathrm{ArH}), 7.53(1 \mathrm{H}, \mathrm{dd}, J=2.4$ and $9.0 \mathrm{~Hz}, \mathrm{ArHs}), 7.20(1 \mathrm{H}, \mathrm{d}$, $\left.J=15.0 \mathrm{~Hz}, \mathrm{H}_{\alpha \beta}\right), 6.91(1 \mathrm{H}, \mathrm{d}, J=9.0 \mathrm{~Hz}, \mathrm{ArH}), 6.45(1 \mathrm{H}, \mathrm{s}$, pyrrole- $\mathrm{H}), 2.21\left(3 \mathrm{H}, \mathrm{s}, \mathrm{CH}_{3}\right), 2.07(3 \mathrm{H}, \mathrm{s}$, $\left.\mathrm{CH}_{3}\right) ;{ }^{13} \mathrm{C} \mathrm{NMR}\left(75 \mathrm{MHz}, \mathrm{CDCl}_{3}\right): \delta_{\mathrm{C}} 192.6,162.5,148.9,139.9,138.9,138.3,135.9,134.3,131.7,131.6$, 130.7, 123.8, 123.4, 121.7, 120.6, 118.7, 114.1, 110.3, 105.4, 12.9, 11.3. $\mathrm{m} / \mathrm{z}$ (ESI-HRMS) found 441.0278, calcd for $\mathrm{C}_{21} \mathrm{H}_{18} \mathrm{~N}_{2} \mathrm{O}_{4} \mathrm{Br}$ : $441.0444[\mathrm{M}+\mathrm{H}]^{+}$. Anal. Found: $\mathrm{C}, 57.66 ; \mathrm{H}, 4.16 ; \mathrm{N}, 6.10 \%$. Anal. calcd. $\mathrm{C}_{21} \mathrm{H}_{17} \mathrm{~N}_{2} \mathrm{O}_{4} \mathrm{Br}$ : C, 57.16; $\mathrm{H}, 3.88 ; \mathrm{N}, 6.35 \%$. 
(E)-1-(5-Bromo-2-hydroxyphenyl)-3-(1-(4-methoxyphenyl)-2,5-dimethyl-1H-pyrrol-3-yl)prop-2-en-1-one 10j, Yellow crystalline solid. Yield $=14 \%$. m.p. $138{ }^{\circ} \mathrm{C}-140{ }^{\circ} \mathrm{C} .{ }^{1} \mathrm{H}$ NMR $\left(400 \mathrm{MHz}, \mathrm{CDCl}_{3}\right): \delta_{\mathrm{H}} 13.3(1 \mathrm{H}, \mathrm{s}$, $\mathrm{OH}), 8.07\left(1 \mathrm{H}, \mathrm{d}, J=14.8 \mathrm{~Hz}, \mathrm{H}_{\alpha \beta}\right), 8.00(1 \mathrm{H}, \mathrm{d}, J=2.4 \mathrm{~Hz}, \mathrm{ArH}), 7.51(1 \mathrm{H}, \mathrm{dd}, J=2.4$ and $8.8 \mathrm{~Hz}$, $\mathrm{ArH}), 7.15\left(1 \mathrm{H}, \mathrm{d}, J=14.8 \mathrm{~Hz}, \mathrm{H}_{\alpha \beta}\right), 7.12(2 \mathrm{H}, \mathrm{d}, J=8.8 \mathrm{~Hz}, \mathrm{ArHs}), 7.01(2 \mathrm{H}, \mathrm{d}, J=8.8 \mathrm{~Hz}, \mathrm{ArHs})$, $6.90(1 \mathrm{H}, \mathrm{d}, J=8.8 \mathrm{~Hz}, \mathrm{ArH}), 6.38\left(1 \mathrm{H}, \mathrm{s}\right.$, pyrrole-H), $3.87\left(3 \mathrm{H}, \mathrm{s}, \mathrm{OCH}_{3}\right), 2.17\left(3 \mathrm{H}, \mathrm{s}, \mathrm{CH}_{3}\right), 2.02(3 \mathrm{H}, \mathrm{s}$, $\left.\mathrm{CH}_{3}\right) .{ }^{13} \mathrm{C}$ NMR $\left(100 \mathrm{MHz}, \mathrm{CDCl}_{3}\right): \delta_{\mathrm{C}} 192.4,162.5,159.8,140.9,138.1,137.3,132.3,131.7,130.2,129.1$, $121.8,120.6,117.8,114.9,112.7,110.0,104.0,55.6,12.8,11.1 . \mathrm{m} / z$ (ESI-HRMS) found 426.0698, calcd for $\mathrm{C}_{22} \mathrm{H}_{21} \mathrm{NO}_{3} \mathrm{Br}: 426.0699[\mathrm{M}+\mathrm{H}]^{+}$.

(E)-1-(5-Bromo-2-hydroxyphenyl)-3-(2,5-dimethyl-1H-pyrrol-3-yl)prop-2-en-1-one 10k, Orange solid. Yield = 9\%. m.p. $88^{\circ} \mathrm{C}-89{ }^{\circ} \mathrm{C} .{ }^{1} \mathrm{H} \mathrm{NMR}\left(400 \mathrm{MHz}, \mathrm{CDCl}_{3}\right): \delta_{\mathrm{H}} 13.3(1 \mathrm{H}, \mathrm{s}, \mathrm{OH}), 8.00-7.97\left(3 \mathrm{H}, \mathrm{m}, \mathrm{ArHs}, \mathrm{H}_{\alpha \beta}\right)$, $7.50(1 \mathrm{H}, \mathrm{dd}, J=2.4$ and $8.8 \mathrm{~Hz}, \mathrm{ArH}), 7.08\left(1 \mathrm{H}, \mathrm{d}, J=14.8 \mathrm{~Hz}, \mathrm{H}_{\alpha \beta}\right), 6.89(1 \mathrm{H}, \mathrm{d}, J=8.8 \mathrm{~Hz}, \mathrm{ArH}), 6.22$ $\left(1 \mathrm{H}, \mathrm{s}\right.$, pyrrole-H) $2.39\left(3 \mathrm{H}, \mathrm{s}, \mathrm{CH}_{3}\right), 2.25\left(3 \mathrm{H}, \mathrm{s}, \mathrm{CH}_{3}\right) .{ }^{13} \mathrm{C} \mathrm{NMR}\left(100 \mathrm{MHz}, \mathrm{CDCl}_{3}\right): \delta_{\mathrm{C}} 192.7,162.3$, 140.6, 137.9, 134.6, 131.6, 128.9, 121.8, 120.6, 118.6, 112.9, 110.2, 104.2, 12.9, 11.5. m/z (ESI-HRMS) found 318.0144, calcd for $\mathrm{C}_{15} \mathrm{H}_{13} \mathrm{NO}_{2} \mathrm{Br}$ : $318.0130[\mathrm{M}-\mathrm{H}]^{+}$. Anal. Found: C, 55.69; H, 2.48; N, 3.44\%; Anal. calcd. $\mathrm{C}_{15} \mathrm{H}_{14} \mathrm{NO}_{2} \mathrm{Br} \cdot \mathrm{CH}_{3} \mathrm{OH}$ : C, 55.37; $\mathrm{H}, 4.80 ; \mathrm{N}, 4.17 \%$.

(E)-1-(5-Bromo-2-hydroxyphenyl)-3-(1-cyclohexyl-2,5-dimethyl-1H-pyrrol-3-yl)prop-2-en-1-one 101, Yellow solid. Yield $=42 \%$. m.p. $73{ }^{\circ} \mathrm{C}-76{ }^{\circ} \mathrm{C} .{ }^{1} \mathrm{H}$ NMR $(400 \mathrm{MHz}, \mathrm{CDCl} 3): \delta_{\mathrm{H}} 13.4(1 \mathrm{H}, \mathrm{s}, \mathrm{OH}), 8.03(1 \mathrm{H}, \mathrm{d}$, $\left.J=14.4 \mathrm{~Hz}, \mathrm{H}_{\alpha \beta}\right), 7.96(1 \mathrm{H}, \mathrm{d}, J=2.4 \mathrm{~Hz}, \mathrm{ArH}), 7.49(1 \mathrm{H}, \mathrm{dd}, J=2.4$ and $9.2 \mathrm{~Hz}, \mathrm{ArH}), 7.07(1 \mathrm{H}, \mathrm{d}$, $\left.J=14.4 \mathrm{~Hz}, \mathrm{H}_{\alpha \beta}\right), 6.88(1 \mathrm{H}, \mathrm{d}, J=8.8 \mathrm{~Hz}, \mathrm{ArH}), 6.27(1 \mathrm{H}, \mathrm{s}$, pyrrole-H), 4.00-3.92 (1H, m, cyclic-H), $2.44\left(3 \mathrm{H}, \mathrm{s}, \mathrm{CH}_{3}\right), 2.33\left(3 \mathrm{H}, \mathrm{s}, \mathrm{CH}_{3}\right), 2.00-1.75\left(7 \mathrm{H}, \mathrm{m}\right.$, cyclic-Hs), $1.45-1.20(3 \mathrm{H}, \mathrm{m}, \mathrm{cyclic}-\mathrm{Hs}) .{ }^{13} \mathrm{C}$ NMR $\left(100 \mathrm{MHz}, \mathrm{CDCl}_{3}\right): \delta_{\mathrm{C}} 192.6,162.5,140.7,137.9,136.2,131.5,131.1,122.0,120.4,112.0,110.2,105.5$, 57.4, 32.3 (2C), 26.4, 25.9, 14.3, 11.8. $\mathrm{m} / \mathrm{z}$ (ESI-HRMS) found 402.1061, calcd for $\mathrm{C}_{21} \mathrm{H}_{25} \mathrm{NO}_{2} \mathrm{Br}: 402.1063$ $[\mathrm{M}+\mathrm{H}]^{+}$. Anal. Found: $\mathrm{C}, 62.08 ; \mathrm{H}, 5.06 ; \mathrm{N}, 3.44 \%$; Anal. calcd. $\mathrm{C}_{21} \mathrm{H}_{24} \mathrm{NO}_{2} \mathrm{Br}: \mathrm{C}, 62.69 ; \mathrm{H}, 6.01$; $\mathrm{N}, 3.48 \%$.

(E)-1-(5-Bromo-2-hydroxyphenyl)-3-(1H-imidazol-4-yl)prop-2-en-1-one 11a. Yellow solid. Yield = 9\%. m.p. $116^{\circ} \mathrm{C}-120{ }^{\circ} \mathrm{C} .{ }^{1} \mathrm{H}$ NMR $\left(600 \mathrm{MHz}, \mathrm{DMSO}-d_{6}\right): \delta_{\mathrm{H}} 12.3(1 \mathrm{H}, \mathrm{s}, \mathrm{OH}), 8.01(1 \mathrm{H}, \mathrm{d}, J=2.4 \mathrm{~Hz}, \mathrm{ArH}), 7.88$ $\left(1 \mathrm{H}, \mathrm{s}\right.$, imidazole-H), $7.73\left(1 \mathrm{H}, \mathrm{s}\right.$, imidazole-H), $7.70\left(1 \mathrm{H}, \mathrm{d}, J=15.0 \mathrm{~Hz}, \mathrm{H}_{\alpha \beta}\right), 7.64-7.61(2 \mathrm{H}, \mathrm{m}, \mathrm{ArH}$, $\left.\mathrm{H}_{\alpha \beta}\right), 6.94(1 \mathrm{H}, \mathrm{d}, J=8.4 \mathrm{~Hz}, \mathrm{ArH}) ;{ }^{13} \mathrm{C} \mathrm{NMR}\left(150 \mathrm{MHz}, \mathrm{DMSO}-d_{6}\right): \delta_{\mathrm{C}} 191.9,159.9,138.5,137.7,136.7$, 131.9 (2C), 123.4, 120.1 (2C), 117.8, 110.13. m/z (ESI-HRMS) found 292.9914, calcd for $\mathrm{C}_{12} \mathrm{H}_{10} \mathrm{~N}_{2} \mathrm{O}_{2} \mathrm{Br}$ : 292.9920 [M+ H] $]^{+}$. Anal. Found: C, 49.99; H, 3.30; N, 9.76. Anal. calcd. $\mathrm{C}_{12} \mathrm{H}_{9} \mathrm{~N}_{2} \mathrm{O}_{2} \mathrm{Br}: \mathrm{C}, 49.17 ; \mathrm{H}$, $3.09 ; \mathrm{N}, 9.56 \%$.

(E)-3-(2,5-Dimethyl-1-(4-nitrophenyl)-1H-pyrrol-3-yl)-1-phenylprop-2-en-1-one, 12, Yellow crystalline solid. Yield $=65 \%$. m.p. $169{ }^{\circ} \mathrm{C}-174{ }^{\circ} \mathrm{C} .{ }^{1} \mathrm{H} \mathrm{NMR}\left(300 \mathrm{MHz}, \mathrm{CDCl}_{3}\right): \delta_{\mathrm{H}} 8.39(2 \mathrm{H}, \mathrm{d}, J=9.0 \mathrm{~Hz}, \mathrm{ArHs}), 8.03-$ $8.00(2 \mathrm{H}, \mathrm{m}, \mathrm{ArHs}), 7.89\left(1 \mathrm{H}, \mathrm{d}, J=15.3 \mathrm{~Hz}, \mathrm{H}_{\alpha \beta}\right), 7.57-7.46(3 \mathrm{H}, \mathrm{m}, \mathrm{ArHs}), 7.40(2 \mathrm{H}, \mathrm{d}, J=8.7 \mathrm{~Hz}$, ArHs), $7.21\left(1 \mathrm{H}, \mathrm{d}, J=15.0 \mathrm{~Hz}, \mathrm{H}_{\alpha \beta}\right), 6.39(1 \mathrm{H}, \mathrm{s}$, pyrrole- $\mathrm{H}), 2.18\left(3 \mathrm{H}, \mathrm{s}, \mathrm{CH}_{3}\right), 2.07\left(3 \mathrm{H}, \mathrm{s}, \mathrm{CH}_{3}\right) .{ }^{13} \mathrm{C}$ NMR $\left(75 \mathrm{MHz}_{,} \mathrm{CDCl}_{3}\right): \delta_{\mathrm{C}} 190.7,147.6,143.6,139.2,137.9,134.1,132.4,130.9,129.2,128.6,128.4,125.1$, $118.9,117.4,105.7,13.1,11.3 . \mathrm{m} / \mathrm{z}$ (ESI-HRMS) found 347.1392, calcd for $\mathrm{C}_{21} \mathrm{H}_{19} \mathrm{~N}_{2} \mathrm{O}_{3}: 347.1390[\mathrm{M}+$ $\mathrm{H}^{+}$. Anal. Found: C, 72.30; H, 6.22; N, 7.44\%. Anal. calcd. $\mathrm{C}_{21} \mathrm{H}_{18} \mathrm{~N}_{2} \mathrm{O}_{3}: \mathrm{C}, 72.82 ; \mathrm{H}, 5.24 ; \mathrm{N}, 8.09 \%$.

(E)-3-(2,5-Dimethyl-1-(4-nitrophenyl)-1H-pyrrol-3-yl)-1-(p-tolyl)prop-2-en-1-one, 13, Yellow solid. Yield = $34 \%$. m.p. $172{ }^{\circ} \mathrm{C}-173{ }^{\circ} \mathrm{C} .{ }^{1} \mathrm{H}$ NMR $\left(400 \mathrm{MHz}, \mathrm{CDCl}_{3}\right): \delta_{\mathrm{H}} 8.39(2 \mathrm{H}, \mathrm{d}, J=8.8 \mathrm{~Hz}, \mathrm{ArHs}), 7.92(2 \mathrm{H}, \mathrm{d}$, $J=8.4 \mathrm{~Hz}, \mathrm{ArHs}), 7.88\left(1 \mathrm{H}, \mathrm{d}, J=15.2 \mathrm{~Hz}, \mathrm{H}_{\alpha \beta}\right), 7.41(2 \mathrm{H}, \mathrm{d}, J=8.8 \mathrm{~Hz}, \mathrm{ArHs}), 7.28(2 \mathrm{H}, \mathrm{d}, J=8 \mathrm{~Hz}$, ArHs), $7.21\left(1 \mathrm{H}, \mathrm{d}, J=15.2 \mathrm{~Hz}, \mathrm{H}_{\alpha \beta}\right), 6.40\left(1 \mathrm{H}, \mathrm{s}\right.$, pyrrole-H), $2.43\left(3 \mathrm{H}, \mathrm{s}, \mathrm{CH}_{3}\right), 2.18\left(3 \mathrm{H}_{,}, \mathrm{s}, \mathrm{CH}_{3}\right)$, $2.06\left(3 \mathrm{H}, \mathrm{s}, \mathrm{CH}_{3}\right) .{ }^{13} \mathrm{C} \mathrm{NMR}\left(75 \mathrm{MHz} \mathrm{CDCl}_{3}\right): \delta_{\mathrm{C}} 190.2,147.5,143.7,143.1,137.4,136.6,133.9,130.8$, $129.3,129.2,128.6,125.0,118.9,117.4,105.7,21.9,13.1,11.3 . \mathrm{m} / z$ (ESI-HRMS) found 361.1552, calcd for $\mathrm{C}_{22} \mathrm{H}_{21} \mathrm{~N}_{2} \mathrm{O}_{3}$ : $361.1547[\mathrm{M}+\mathrm{H}]^{+}$. Anal. Found: $\mathrm{C}, 73.08 ; \mathrm{H}, 5.86 ; \mathrm{N}, 7.43 \%$. Anal. calcd. $\mathrm{C}_{22} \mathrm{H}_{20} \mathrm{~N}_{2} \mathrm{O}_{3}$ : C, 73.32; H, 5.59; N, 7.77\%. 
(E)-3-(2,5-Dimethyl-1-(4-nitrophenyl)-1H-pyrrol-3-yl)-1-(4-fluorophenyl)prop-2-en-1-one, 14, Yellow solid. Yield $=86 \%$. m.p. $160{ }^{\circ} \mathrm{C}-164{ }^{\circ} \mathrm{C} .{ }^{1} \mathrm{H} \mathrm{NMR}\left(300 \mathrm{MHz}, \mathrm{CDCl}_{3}\right): \delta_{\mathrm{H}} 8.39(2 \mathrm{H}, \mathrm{d}, J=9.0 \mathrm{~Hz}, \mathrm{ArHs})$, 8.07-8.02 (2H, m, ArHs), $7.89\left(1 \mathrm{H}, \mathrm{d}, J=15.3 \mathrm{~Hz}, \mathrm{H}_{\alpha \beta}\right), 7.41(2 \mathrm{H}, \mathrm{d}, J=9.0 \mathrm{~Hz}, \mathrm{ArHs}), 7.205-7.12$ $\left(3 \mathrm{H}, \mathrm{m}, \mathrm{ArHs}, \mathrm{H}_{\alpha \beta}\right), 6.39\left(1 \mathrm{H}, \mathrm{s}\right.$, pyrrole-H) $2.19\left(3 \mathrm{H}, \mathrm{s}, \mathrm{CH}_{3}\right), 2.07\left(3 \mathrm{H}, \mathrm{s}, \mathrm{CH}_{3}\right) .{ }^{13} \mathrm{C} \mathrm{NMR}(75 \mathrm{MHz}$, $\left.\mathrm{CDCl}_{3}\right): \delta_{\mathrm{C}} 189.1,147.7,143.8,138.3,135.6,134.5,131.1,131.0,129.3,125.2,119.1,117.0,115.9,115.7$, 105.7, 13.2, 11.4. $\mathrm{m} / z$ (ESI-HRMS) found 365.1305, calcd for $\mathrm{C}_{21} \mathrm{H}_{18} \mathrm{~N}_{2} \mathrm{O}_{3} \mathrm{~F}: 365.1296[\mathrm{M}+\mathrm{H}]^{+}$. Anal. Found: C, 69.15; H, 5.24; N, 7.19\%. Anal. calcd. $\mathrm{C}_{21} \mathrm{H}_{17} \mathrm{~N}_{2} \mathrm{O}_{3} \mathrm{~F}: \mathrm{C}, 69.22 ; \mathrm{H}, 4.70 ; \mathrm{N}, 7.69 \%$.

(E)-3-(2,5-Dimethyl-1-(4-nitrophenyl)-1H-pyrrol-3-yl)-1-(4-methoxyphenyl)prop-2-en-1-one, 15, Yellow crystalline solid. Yield $=18 \%$. m.p. $157{ }^{\circ} \mathrm{C}-158{ }^{\circ} \mathrm{C} .{ }^{1} \mathrm{H}$ NMR $\left(300 \mathrm{MHz}, \mathrm{CDCl}_{3}\right): \delta_{\mathrm{H}} 8.39(2 \mathrm{H}$, $\mathrm{d}, J=8.7 \mathrm{~Hz}, \mathrm{ArHs}), 8.04(2 \mathrm{H}, \mathrm{d}, J=9.0 \mathrm{~Hz}, \mathrm{ArHs}), 7.88\left(1 \mathrm{H}, \mathrm{d}, J=15.3 \mathrm{~Hz}, \mathrm{H}_{\alpha \beta}\right), 7.41(2 \mathrm{H}, \mathrm{d}, J=9.0 \mathrm{~Hz}$, ArHs), $7.23\left(1 \mathrm{H}, \mathrm{d}, J=15.3 \mathrm{~Hz}, \mathrm{H}_{\alpha \beta}\right), 6.97(2 \mathrm{H}, \mathrm{d}, J=9.0 \mathrm{~Hz}, \mathrm{ArHs}), 6.39$ (1H, s, pyrrole-H), 3.89 (3H, s, $\left.\mathrm{CH}_{3}\right), 2.18\left(3 \mathrm{H}, \mathrm{s}, \mathrm{CH}_{3}\right), 2.07\left(3 \mathrm{H}, \mathrm{s}, \mathrm{CH}_{3}\right) .{ }^{13} \mathrm{C} \mathrm{NMR}\left(75 \mathrm{MHz}_{2} \mathrm{CDCl}_{3}\right): \delta_{\mathrm{C}} 189.0,163.1,147.4,143.7$, 137.0, 133.8, 131.9, 130.7, 130.6, 129.1, 125.0, 118.9, 117.1, 113.8, 105.5, 55.5, 13.0, 11.5. m/z (ESI-HRMS) found 377.1502, calcd for $\mathrm{C}_{22} \mathrm{H}_{21} \mathrm{~N}_{2} \mathrm{O}_{4}$ : $377.1496[\mathrm{M}+\mathrm{H}]^{+}$. Anal. Found: $\mathrm{C}, 68.91 ; \mathrm{H}, 5.73 ; \mathrm{N}, 7.23 \%$. Anal. calcd. $\mathrm{C}_{22} \mathrm{H}_{20} \mathrm{~N}_{2} \mathrm{O}_{4} \cdot 0.5 \mathrm{CH}_{3} \mathrm{OH}$ : C, 68.86; $\mathrm{H}, 5.65 ; 7.14 \%$.

(E)-1-(2,4-Dimethoxyphenyl)-3-(2,5-dimethyl-1-(4-nitrophenyl)-1H-pyrrol-3-yl)prop-2-en-1-one 16. Yellow crystalline solid. Yield $=21 \%$. m.p. $146{ }^{\circ} \mathrm{C}-149{ }^{\circ} \mathrm{C} .{ }^{1} \mathrm{H}$ NMR $\left(600 \mathrm{MHz}, \mathrm{CDCl}_{3}\right): \delta_{\mathrm{H}} 8.38(2 \mathrm{H}, \mathrm{d}$, $J=8.4 \mathrm{~Hz}$, ArHs), 7.75-7.72 (2H, m, ArH, $\left.\mathrm{H}_{\alpha \beta}\right), 7.40(2 \mathrm{H}, \mathrm{d}, J=9.0 \mathrm{~Hz}, \mathrm{ArHs}), 7.16(1 \mathrm{H}, \mathrm{d}, J=15.6 \mathrm{~Hz}$, $\left.\mathrm{H}_{\alpha \beta}\right), 6.55(1 \mathrm{H}, \mathrm{dd}, J=2.4$ and $9.0 \mathrm{~Hz}, \mathrm{ArH}), 6.50(1 \mathrm{H}, \mathrm{d}, J=2.4 \mathrm{~Hz}, \mathrm{ArH}), 6.32(1 \mathrm{H}, \mathrm{s}$, pyrrole-H), 3.90 $\left(3 \mathrm{H}, \mathrm{s}, \mathrm{CH}_{3}\right), 3.87\left(3 \mathrm{H}, \mathrm{s}, \mathrm{CH}_{3}\right), 2.15\left(3 \mathrm{H}, \mathrm{s}, \mathrm{CH}_{3}\right), 2.053\left(3 \mathrm{H}, \mathrm{s}, \mathrm{CH}_{3}\right) .{ }^{13} \mathrm{C} \mathrm{NMR}\left(75 \mathrm{MHz}, \mathrm{CDCl}_{3}\right): \delta_{\mathrm{C}}$ 191.0, 163.8, 160.2, 147.5, 143.8, 135.6, 133.2, 132.7, 130.5, 129.2, 125.0, 123.2, 122.9, 119.1, 105.8, 105.1, 98.9, 55.9, 55.7, 13.1, 11.3. $\mathrm{m} / \mathrm{z}$ (ESI-HRMS) found 407.1608, calcd for $\mathrm{C}_{23} \mathrm{H}_{23} \mathrm{~N}_{2} \mathrm{O}_{5}: 407.1601[\mathrm{M}+\mathrm{H}]^{+}$. Anal. Found C, 67.99; H, 5.84; N, 6.86\%; Anal. calcd. $\mathrm{C}_{23} \mathrm{H}_{22} \mathrm{~N}_{2} \mathrm{O}_{5}: \mathrm{C}, 67.41 ; \mathrm{H}, 5.61 ; \mathrm{N}, 6.61 \%$.

(E)-3-(2,5-Dimethyl-1-(4-nitrophenyl)-1H-pyrrol-3-yl)-1-(4-nitrophenyl)prop-2-en-1-one, 17, Reddish solid. Yield $=5 \%$. m.p. $175^{\circ} \mathrm{C}-178^{\circ} \mathrm{C} .{ }^{1} \mathrm{H}$ NMR $\left(400 \mathrm{MHz}, \mathrm{CDCl}_{3}\right): \delta_{\mathrm{H}} 8.41(2 \mathrm{H}, \mathrm{d}, J=8.8 \mathrm{~Hz}, \mathrm{ArHs}), 8.33(2 \mathrm{H}$, $\mathrm{d}, J=8.4 \mathrm{~Hz}, \mathrm{ArHs}), 8.13(2 \mathrm{H}, \mathrm{d}, J=8.8 \mathrm{~Hz}, \mathrm{ArHs}), 7.93\left(1 \mathrm{H}, \mathrm{d}, J=15.2 \mathrm{~Hz}, \mathrm{H}_{\alpha \beta}\right), 7.42(2 \mathrm{H}, \mathrm{d}, J=8.8 \mathrm{~Hz}$, ArHs), $7.14\left(1 \mathrm{H}, \mathrm{d}, J=15.2 \mathrm{~Hz}, \mathrm{H}_{\alpha \beta}\right), 6.40\left(1 \mathrm{H}, \mathrm{s}\right.$, pyrrole-H), $2.20\left(3 \mathrm{H}, \mathrm{s}, \mathrm{CH}_{3}\right), 2.07\left(3 \mathrm{H}, \mathrm{s}, \mathrm{CH}_{3}\right) .{ }^{13} \mathrm{C}$ NMR $\left(100 \mathrm{MHz}, \mathrm{CDCl}_{3}\right): \delta_{\mathrm{C}} 188.9,149.9,147.7,144.3,143.4,139.9,135.3,131.4(2 \mathrm{C}), 129.3,129.2,125.2$, $123.9,118.9,116.5,105.6,13.1,11.4 . \mathrm{m} / \mathrm{z}$ (ESI-HRMS) found 392.1246, calcd for $\mathrm{C}_{21} \mathrm{H}_{18} \mathrm{~N}_{3} \mathrm{O}_{5}: 392.1241$ $[\mathrm{M}+\mathrm{H}]^{+}$. Anal. Found: $\mathrm{C}, 63.30 ; \mathrm{H}, 5.00 ; \mathrm{N}, 9.29 \%$. Anal. calcd. $\mathrm{C}_{21} \mathrm{H}_{17} \mathrm{~N}_{3} \mathrm{O}_{5} \cdot 0.5 \mathrm{CH}_{3} \mathrm{COCH}_{2} \mathrm{CH}_{3}: \mathrm{C}_{\text {, }}$ $63.44 ; \mathrm{H}, 4.86 ; \mathrm{N}, 9.65 \%$.

(E)-1-(5-Chloro-2-hydroxyphenyl)-3-(2,5-dimethyl-1-(3-(trifluoromethyl)phenyl)-1H-pyrrol-3-yl)prop-2-en-1-one 18. Yellow crystalline solid. Yield $=87 \%$. m.p. $110{ }^{\circ} \mathrm{C}-112{ }^{\circ} \mathrm{C} .{ }^{1} \mathrm{H}$ NMR $\left(400 \mathrm{MHz}, \mathrm{CDCl}_{3}\right): \delta_{\mathrm{H}} 13.2$ $(1 \mathrm{H}, \mathrm{s}, \mathrm{OH}), 8.04(1 \mathrm{H}, \mathrm{d}, J=2.4 \mathrm{~Hz}, \mathrm{ArH}), 7.76(1 \mathrm{H}, \mathrm{d}, J=7.6 \mathrm{~Hz}, \mathrm{ArH}), 7.70-7.66(1 \mathrm{H}, \mathrm{m}, \mathrm{ArH})$, 7.52-7.49 (1H, m, ArH), 7.45-7.42 (1H, m, ArH), 7.40 (1H, dd, J = 2.4 and 9.2 Hz, ArH), $7.19(1 \mathrm{H}, \mathrm{d}$, $\left.J=14.8 \mathrm{~Hz}, \mathrm{H}_{\alpha \beta}\right), 6.95(1 \mathrm{H}, \mathrm{d}, J=8.8 \mathrm{~Hz}, \mathrm{ArH}), 6.42\left(1 \mathrm{H}, \mathrm{s}\right.$, pyrrole-H), $2.19\left(3 \mathrm{H}, \mathrm{s}, \mathrm{CH}_{3}\right), 2.05(3 \mathrm{H}$, $\left.\mathrm{s}, \mathrm{CH}_{3}\right) .{ }^{13} \mathrm{C} \mathrm{NMR}\left(100 \mathrm{MHz}, \mathrm{CDCl}_{3}\right): \delta_{\mathrm{C}} 192.7,162.1,140.1,138.4,136.2,135.9,135.4,132.3,131.7$, 131.6, 130.4, 128.7, 125.7, 125.1, 123.3, 121.2, 120.2, 118.5, 113.8, 105.1, 12.9, 11.2. $\mathrm{m} / \mathrm{z}$ (ESI-HRMS) found 420.0945, calcd for $\mathrm{C}_{22} \mathrm{H}_{17} \mathrm{NO}_{2} \mathrm{ClF}_{3}$ : $420.0948[\mathrm{M}+\mathrm{H}]^{+}$. Anal. Found: $\mathrm{C}, 62.34 ; \mathrm{H}, 4.28 ; \mathrm{N}, 2.94 \%$; Anal. calcd. $\mathrm{C}_{22} \mathrm{H}_{17} \mathrm{NO}_{2} \mathrm{ClF}_{3} \cdot 0.5 \mathrm{CH}_{3} \mathrm{CH}_{2} \mathrm{OH}$ : C, 62.38; $\mathrm{H}, 4.55 ; \mathrm{N}, 3.16 \%$.

(E)-1-(5-Chloro-2-hydroxyphenyl)-3-(2,5-dimethyl-1-(3-nitrophenyl)-1H-pyrrol-3-yl)prop-2-en-1-one 19, Yellow crystalline solid. Yield $=89 \%$. m.p. $71{ }^{\circ} \mathrm{C}-74{ }^{\circ} \mathrm{C}$. IR $\left(\mathrm{cm}^{-1}\right): 1630(\mathrm{C}=\mathrm{O}), 1527(\mathrm{C}=\mathrm{C}) .{ }^{1} \mathrm{H}$ NMR $\left(300 \mathrm{MHz}, \mathrm{CDCl}_{3}\right): \delta_{\mathrm{H}} 13.2(1 \mathrm{H}, \mathrm{s}, \mathrm{OH}), 8.38-8.34(1 \mathrm{H}, \mathrm{m}, \mathrm{ArH}), 8.14-8.13(1 \mathrm{H}, \mathrm{m}, \mathrm{ArH}), 8.03(1 \mathrm{H}, \mathrm{d}$, $\left.J=15.0 \mathrm{~Hz}, \mathrm{H}_{\alpha \beta}\right), 7.86(1 \mathrm{H}, \mathrm{d}, J=2.7 \mathrm{~Hz}, \mathrm{ArH}), 7.77-7.72(1 \mathrm{H}, \mathrm{m}, \mathrm{ArH}), 7.61-7.58(1 \mathrm{H}, \mathrm{m}, \mathrm{ArH}), 7.40$ $(1 \mathrm{H}, \mathrm{dd}, J=2.4$ and $8.7 \mathrm{~Hz}, \mathrm{ArH}), 7.20\left(1 \mathrm{H}, \mathrm{d}, J=14.7 \mathrm{~Hz}, \mathrm{H}_{\alpha \beta}\right), 6.96(1 \mathrm{H}, \mathrm{d}, J=9.0 \mathrm{~Hz}, \mathrm{ArH}), 6.44(1 \mathrm{H}$, s, pyrrole-H), $2.19\left(3 \mathrm{H}, \mathrm{s}, \mathrm{CH}_{3}\right), 2.07\left(3 \mathrm{H}, \mathrm{s}, \mathrm{CH}_{3}\right) .{ }^{13} \mathrm{C} \mathrm{NMR}\left(75 \mathrm{MHz}, \mathrm{CDCl}_{3}\right): \delta_{\mathrm{C}} 192.8,162.1,148.9$, $139.8,138.9,135.9,135.5,134.3,131.5,130.7,128.7,123.7,123.3$ (2C), 121.1, 120.2, 118.7, 114.1, 105.3, 13.0, 
11.3. $\mathrm{m} / \mathrm{z}$ (ESI-HRMS) found 397.0947, calcd for $\mathrm{C}_{21} \mathrm{H}_{18} \mathrm{~N}_{2} \mathrm{O}_{4} \mathrm{Cl}: 397.0955[\mathrm{M}+\mathrm{H}]^{+}$. Anal. Found: $\mathrm{C}$, 63.74; $\mathrm{H}, 5.23 ; \mathrm{N}, 6.62 \%$; Anal. calcd. $\mathrm{C}_{21} \mathrm{H}_{17} \mathrm{~N}_{2} \mathrm{O}_{4} \mathrm{Cl} \cdot 0.4 \mathrm{CH}_{3} \mathrm{CH}_{2} \mathrm{OH}$ : C, 63.06; $\mathrm{H}, 4.71 ; \mathrm{N}, 6.75 \%$.

\subsection{X-ray Crystallographic Analysis}

A single crystal was covered in a small amount of paratone $\mathrm{N}$ oil and mounted on a MiTeGen microloop. X-ray intensity data were collected at $100 \mathrm{~K}$ on a Bruker DUO APEX CCD with graphite monochromated Mo radiation $(\lambda=0.71073)$. The detector to crystal distance was $60 \mathrm{~mm}$. Data were collected using phi and omega scans and were scaled and reduced using the APEX III software suite (Bruker SAINT). The structure was solved using SHELXT 2018/2 (Sheldrick, 2018) and refined using SHELXL-2018/3 (Sheldrick, 2018) [52]. Hydrogen atoms were placed in calculated positions and refined using the riding model. The program X-SEED [53], an interface to SHELX, was used during the structure solution and refinement. The accession code for 10e is (CCDC 1991652). The supplementary information contains crystallographic data for compound 10e. The data can be obtained free of charge from The Cambridge Crystallographic Data Centre on http://www.ccdc.cam.ac.uk/conts/retrieving. html or The Director, CCDC, 12, Union Road, Cambridge CB2 1EZ, UK; fax: +44 1223 336033; or deposit@ccdc.cam.ac.uk.

\subsection{In Vitro Antitrypanosomal Assay}

Trypanosoma brucei brucei 427 trypomastigotes were cultured in Iscove's Modified Dulbecco's medium (IMDM, Lonza, Basel Switzerland) and supplemented with 10\% fetal calf serum, HMI-9 supplement [54], hypoxanthine and penicillin/streptomycin at $37^{\circ} \mathrm{C}$ in a $5 \% \mathrm{CO}_{2}$ incubator. Serial dilutions of test compounds were incubated with the parasites in 96-well plates for $24 \mathrm{~h}$, and residual parasite viability in the wells determined by adding $20 \mu \mathrm{L}$ resazurin toxicology reagent (Sigma-Aldrich) and incubating for an additional $24 \mathrm{~h}$. Reduction of resazurin to resorufin by viable parasites was assessed by fluorescence readings (excitation $560 \mathrm{~nm}$, emission $590 \mathrm{~nm}$ ) in a Spectramax M3 plate reader (Molecular Devices, San Jose, CA, USA). Fluorescence readings were converted to \% parasite viability relative to the average readings obtained from untreated control wells. $\mathrm{IC}_{50}$ values were determined by plotting \% viability vs. $\log [$ compound] and performing non-linear regression using GraphPad Prism (v. 5.02) software [55].

\subsection{In Vitro Cytotoxicity Assay}

HeLa cells (Cellonex, Johannesburg, South Africa) seeded in 96-well plates were incubated with $20 \mu \mathrm{M}$ test compounds and cell viability assessed with resazurin, as previously described [56]. Fluorescence readings (excitation $560 \mathrm{~nm}$, emission $590 \mathrm{~nm}$ ) obtained for the individual wells were converted to \% cell viability relative to the average readings obtained from untreated control wells. Plots of $\%$ cell viability vs. $\log$ [compound] were used to determine $\mathrm{IC}_{50}$ values by non-linear regression using GraphPad Prism (v. 5.02) [56].

\section{Conclusions}

In this study, we presented a series of arylpyrrole-chalcone derivatives, which were obtained in yields ranging from poor to excellent. Analysis of the NMR and crystal structure of compound 10f confirmed unequivocally that achieved compounds assumed a trans configuration about the double bond of the $\alpha, \beta$-unsaturated carbonyl system. The in vitro bioassays data of resultant compounds suggested that they are generally non-toxic, and displayed weak growth inhibition of HeLa cells at $20 \mu \mathrm{M}$, and none of the compounds inhibited cell viability to $<80 \%$. More importantly, these compounds showed anti-trypanosomal activity, with most active compounds 10e and 10h displaying $\mathrm{IC}_{50}$ values of $<6.0 \mu \mathrm{M}$. Despite modest activity, a combination of electron donating groups on the ring $\mathrm{A}$ and electron withdrawing groups on the $\mathrm{B}$ ring appeared to be required for the activity of these compounds. Compounds lacking the aryl groups on the B ring side of the chalcone scaffold showed no activity at the maximum tested concentration, suggesting that the arylpyrrole moiety is necessary for 
activity, and it is worthy of further exploitation for the development of alternative compounds for the treatment of infections caused by trypanosomes.

Supplementary Materials: The following are available online (Synthesis of arylpyrrole intermediates, compound 8a-1, X-ray crystallographic data of compound 10e and selected ${ }^{1} \mathrm{H}$ and ${ }^{13} \mathrm{C}$ NMR spectra of some compounds).

Author Contributions: A.I.Z. synthesized target chalcones and collection of experimental data. O.O.O. contributed some of the starting arylpyrroles. C.K. contributed on some single crystal X-ray data. H.C.H. and M.I. coordinated and performed the T. b. brucei and cytotoxicity assays. V.J.S. assisted and coordinated single crystal X-ray diffraction analysis. C.G.L.V. and S.D.K. conceptualized and provided intellectual leadership of the project including supervision. All authors have read and agreed to the published version of the manuscript

Funding: The research was funded by the Rhodes University Sandisa Imbewu (SDK, CGLV, and HCH) and Thuthuka National Research Foundation (SDK), grant number 87894 and the APC was funded by Rhodes University. The bioassay component of the project was funded by the South African Medical Research Council (MRC) with funds from National Treasury under its Economic Competitiveness and Support Package.

Acknowledgments: AIZ gratefully acknowledged financial support from the National Research Foundation Innovation. OOO and CK were supported by Rhodes University through Sandisa Imbewu. Our great appreciation from the Stellenbosch University Central Analytical Facility (CAF) for mass spectrometric analysis.

Conflicts of Interest: The authors declare no conflict of interest.

\section{References}

1. Deeks, E.D.; Lyseng-Williamson, K.A. Fexinidazole in human African trypanosomiasis: A profile of its use. Drugs Ther. Perspect. 2019, 35, 529-535. [CrossRef]

2. Berninger, M.; Schunidt, I.; Ponte-Sucre, A.; Holzgrabe, U. Novel lead compounds in pre-clinical development against African sleeping sickness. Med. Chem. Commun. 2017, 8, 1872-1890. [CrossRef] [PubMed]

3. Njogore, M.; Njuguna, N.M.; Mutai, P.; Ongarora, D.S.B.; Smith, P.W.; Chibale, K. Recent approaches to chemical discovery and development against malaria and the neglected tropical diseases human African trypanosomiasis and schistosomiasis. Chem. Rev. 2014, 114, 11138-11163. [CrossRef] [PubMed]

4. Castillo-Acosta, V.; Ruiz-Pérez, L.; Etxebarria, J.; Reichardt, N.; Navarro, M.; Igarashi, Y.; Liekens, S.; Balzarini, J.; González-Pacanowska, D. Open source drug discovery with the malaria box compound collection for neglected diseases and beyond. PLoS Pathog. 2016, 12, e1005851.

5. Burrows, J.N.; Elliott, R.L.; Kaneko, T.; Mowbray, C.E.; Waterson, D. The role of modern drug discovery in the fight against neglected and tropical diseases. Med. Chem. Comm. 2014, 5, 688-700. [CrossRef]

6. Kwofie, K.D.; Tung, N.H.; Suzuki-Ohashi, M.; Amoa-Bosompem, M.; Adegle, R.; Sakyiamah, M.M.; Ayertey, F.; Owusu, K.B.-A.; Tuffour, I.; Atchoglo, P. Antitrypanosomal activities and mechanisms of action of novel tetracyclic iridoids from Morinda lucida benth. Antimicrob. Agents Chemother. 2016, 60, 3283-3290. [CrossRef]

7. WHO. Trypanosomiasis, Human African (Sleeping Sickness). Available online: http://www.who.int/ mediacentre/factsheets/fs259/en (accessed on 1 December 2019).

8. Klung, D.M.; Gelb, M.H.; Pollastri, M.P. Repurposing strategies for tropical disease drug discovery. Bioorg. Med. Chem. Lett. 2016, 26, 2569-2576. [CrossRef]

9. CDC. Parasites African Trypanosomiasis (Also Known as Sleeping Sickness). Available online: https: //www.cdc.gov/parasites/sleepingsickness/ (accessed on 1 December 2019).

10. Franco, J.R.; Simarro, P.P.; Diarra, A.; Jannin, J.G. Epidemiology of human African trypanosomiasis. Clin. Epidemiol. 2014, 6, 257-275.

11. Bouteille, B.; Buguet, A. The detection and treatment of human African trypanosomiasis. Res. Rep. Trop. Med. 2012, 3, 35-45. [CrossRef]

12. Kaiser, M.; Bray, M.A.; Cal, M.; Trunz, B.; Torreele, E.; Brun, R. Anti-trypanosomal activity of fexinidazole, a new oral nitroimidazole drug candidate for treatment of sleeping sickness. Antimicrob. Agents Chemother. 2011, 55, 5602-5608. [CrossRef]

13. Mishina, Y.V.; Krishna, S.; Haynes, R.K.; Meade, J.C. Artemisinins inhibit Trypanosoma cruzi and Trypanosoma brucei rhodisiense in vitro growth. Antimicrob. Agents Chemother. 2007, 51, 1852-1854. [CrossRef] [PubMed]

14. Willyard, C. Putting sleeping sickness to bed. Nat. Med. 2011, 17, 14-17. [CrossRef] [PubMed] 
15. Balasegana, M.; Young, H.; Chappuis, F.; Priotto, G.; Raguenaud, M.E.; Checchi, F. Effectiveness of melarsoprol and eflornithine as first-line regimens for gambiense sleeping sickness in nine Médecins Sans Frontières programmes. Trans. R. Soc. Trop. Med. Hyg. 2009, 103, 280-290. [CrossRef] [PubMed]

16. Yang, P.Y.; Wang, M.; Li, L.; Wu, H.; He, C.Y.; Yao, S.Q. Design, synthesis and biological evaluation of potent azadipeptide nitrile inhibitors and activity-based probes as promising anti-trypanosoma brucei agents. Chem. Eur. J. 2012, 18, 6528-6541. [CrossRef]

17. Giordani, F.; Morrison, L.J.; Rowan, T.G.; De Koning, H.P.; Barrett, M.P. The animal trypanosomiases and their chemotherapy: A review. Parasitology 2016, 143, 1862-1889. [CrossRef]

18. Babokhov, P.; Sanyaolu, A.O.; Oyibo, W.A.; Fagbeno-Beioku, A.F.; Iriemenam, N.C. A current analysis of chemotherapy strategies for the treatment of human African trypanosomiasis: A review. Pathog. Glob. Health 2013, 107, 242-252. [CrossRef]

19. Priotto, G.; Kasparian, S.; Mutombo, W.; Ngovama, D.; Ghorashian, S.; Arnold, U.; Ghabri, S.; Baudin, E.; Buard, V.; Kazadi-Kyanza, S.; et al. Nifurtimox-eflornithine combination therapy for second-stage African Trypanosoma brucei gambiense trypanosomiasis: A multicentre, randomised, phase III, non-inferiority trial. Lancet 2000, 374, 56-64. [CrossRef]

20. Deeks, E.D. Fexinidazole: First global approval. Drugs 2019, 79, 215-220. [CrossRef]

21. Veale, C.G.L.; Laming, D.; Swart, T.; Chibale, K.; Hoppe, H.C. Exploring the antiplasmodial 2-aminopyrridines as potential anti-trypanosomal agents. ChemMedChem 2019, 14, 2034-2041. [CrossRef]

22. Veale, C.G.L.; Hoppe, C. Screening of the Pathogen Box reveals new starting points for anti-trypanosomal drug discovery. MedChem Comm 2019, 9, 2037-2044. [CrossRef]

23. Pedron, J.; Boudo, C.; Bourgeade-Delmas, S.; Sourin-Saquet, A.; Paloque, L.; Rastegari, M.; Abdoulaye, M.; El-Kashef, H.; Bonduelle, C.; Pratviel, G.; et al. Anti-trypanosomatid pharmacomodulation at position 3 of the 8-nitroquinolin-2 $(1 H)$-one scaffold using palladium-catalysed cross-coupling reactions. ChemMedChem 2018, 13, 2217-2228. [CrossRef] [PubMed]

24. Mahapatra, D.K.; Bharti, S.K.; Asati, V. Chalcone scaffolds as anti-infective agents: Structural and molecular target perspectives. Eur. J. Med. Chem. 2015, 101, 496-524. [CrossRef] [PubMed]

25. Zhuang, C.; Zhang, W.; Sheng, C.; Zhang, W.; Xing, C. Chalcone: A privileged structure in medicinal chemistry. Chem. Rev. 2017, 117, 7762-7810. [CrossRef] [PubMed]

26. Rohrmann, E.; Jones, R.G.; Shonle, H.A. The use of chalcones in the synthesis of medicinal intermediates. Am. Chem. Soc. 1944, 66, 1856-1857. [CrossRef]

27. Bano, S.; Javed, K.; Ahmad, S.; Rathish, I.G.; Singh, S.; Chaitanya, M.; Arunasree, K.M. Synthesis of some novel chalcones, flavanones and flavones and evaluation of their anti-inflammatory activity. Eur. J. Med. Chem. 2013, 65, 51-59. [CrossRef]

28. Anandam, R.; Jadav, S.S.; Ala, V.B.; Ahsan, M.J.; Bollikolla, H.B. Synthesis of new C-dimethylated chalcones as potent antitubercular agents. Med. Chem. Res. 2018, 27, 1690-1704. [CrossRef]

29. Ventura, T.L.B.; Calixto, S.D.; Abrahim-Vieira, B.A.; de Souza, A.M.T.; Mello, M.V.P.; Rodrigues, C.R.; Miranda, L.S.M.; de Souza, R.O.C.; Leal, I.C.R.; Lasunskaia, E.B.; et al. Antimycobacterial and anti-inflammatory activities of substituted chalcones focusing on an anti-tuberculosis dual treatment approach. Molecules 2015, 20, 8072-8093. [CrossRef]

30. Syahri, J.; Yuanita, E.; Nurohmah, B.A.; Armunanto, R.; Purwono, B. Chalcone analogue as potent anti-malarial compounds against Plasmodium falciparum: Synthesis, biological evaluation, and docking simulation study. Asian Pac. J. Trop. Biomed. 2017, 7, 675-679. [CrossRef]

31. Sinha, S.; Batovska, D.I.; Medhi, B.; Radotra, B.D.; Bhalla, A.; Markova, N.; Sehgal, R. In vitro anti-malarial efficacy of chalcones: Cytotoxicity profile, mechanism of action and their effect on erythrocytes. Malar. J. 2019, 18, 421-431. [CrossRef]

32. Wang, M.; Qin, H.L.; Leng, J.; Amjad, M.W.; Raja, M.A.G.; Hussain, M.A.; Bukhari, S.N.A. Synthesis and biological evaluation of new tetramethylpyrazine-based chalcone derivatives as potential anti-Alzheimer agents. Chem. Biol. Drug Des. 2018, 92, 1859-1866. [CrossRef]

33. Karthikeyan, C.; Narayana Moorthy, N.S.H.; Ramasamy, S.; Vanam, U.; Manivannan, E.; Karunagaran, D.; Trivedi, P. Advances in chalcones with anticancer activities. Recent Pat. Anti-Cancer Drug Discov. 2015, 10, 97-115. [CrossRef] [PubMed] 
34. Qiao, Z.; Wang, Q.; Zhang, F.; Bowling, T.; Nare, B.; Jacobs, R.T.; Zhang, J.; Ding, D.; Liu, Y.; Zhou, H. Chalcone-benzoxaborole hybrid molecules as potent antitrypanosomal agents. J. Med. Chem. 2012, 55, 3553-3557. [CrossRef] [PubMed]

35. Osório, T.M.; Monache, F.D.; Chiaradia, L.D.; Mascarello, A.; Stumpf, T.R.; Zanetti, C.R.; Silveira, D.B.; Barardi, C.R.M.; de Fatima Albino Smânia, E.; Viancelli, A.; et al. Antibacterial activity of chalcones, hydrazones and oxadiazoles against methicillin-resistant Stapphylococcus aureus. Bioorg. Med. Chem. Lett. 2012, 22, 225-230. [CrossRef] [PubMed]

36. Murugesan, D.; Kaiser, M.; White, K.L.; Norval, S.; Riley, J.; Wyatt, P.G.; Charman, S.A.; Read, K.D.; Yeates, C.; Gilbert, I.H. Structure-activity relationship studies of pyrrolone antimalarial agents. ChemMedChem 2013, 8, 1537-1544. [CrossRef]

37. Patil, S.; Utale, P.; Gholse, S.; Pande, S.; Thakur, S. Synthesis, characterization and antimicrobial activity of 2-hydroxy-5-bromo-4-methoxy-N-(substituted phenyl) chalconeimine. Int. J. Pharm. Res. Sch. 2013, 2, 129-135.

38. Dave, S.S.; Ghatole, A.M.; Rahatgaonkar, A.M.; Chorghade, M.S.; Chauhan, P.; Srivastava, K. Experimental and computational evaluation of new quinolinyl chalcones as potent antiplasmodial agents. Indian J. Chem. Sect. B 2009, 48B, 1780-1793.

39. Cardona, G.W.; Yepes, A.F.; Herrera, R.A. Hybrid molecules: Promising compounds for the development of new treatment against leishmaniasis and Chagas disease. Curr. Med. Chem. 2018, 25, 1-43. [CrossRef]

40. Kerru, N.; Singh, P.; Koorbanally, N.; Raj, R.; Kumar, V. Recent advances (2015-2016) in anticancer hybrids. Eur. J. Med. Chem. 2017, 142, 179-212. [CrossRef]

41. Mishra, S.; Singh, P. Hybrid molecules: The privileged scaffolds for various pharmaceuticals. Eur. J. Med. Chem. 2016, 124, 500-536.

42. Darrell, O.T.; Hulushe, S.T.; Mtshare, T.E.; Beteck, R.M.; Isaacs, M.; Laming, D.; Hoppe, H.C.; Krause, R.W.M.; Khanye, S.D. Synthesis, antiplasmodial and antitrypanosomal evaluation of a series of novel 2-oxoquinoline-based thiosemicarbazone derivatives. S. Afr. J. Chem. 2018, 71, 174-181. [CrossRef]

43. Gumbo, M.; Beteck, R.M.; Mandizvo, T.; Seldon, R.; Warner, D.F.; Hoppe, H.C.; Isaacs, M.; Laming, D.; Tam, C.C.; Cheng, L.W.; et al. Cinnamoyl-Oxaborole Amides: Synthesis and Their in Vitro Biological Activity. Molecules 2018, 23, 2038. [CrossRef] [PubMed]

44. Beteck, R.M.; Isaacs, M.; Hoppe, H.C.; Khanye, S.D. Synthesis, in vitro cytotoxicity and trypanocidal evaluation of novel 1,3,6-substituted non-fluoroquinolones. S. Afr. J. Chem. 2018, 71, 188-195. [CrossRef]

45. Amarnath, V.; Anthony, D.C.; Amarnath, K.; Valentine, W.M.; Wetterau, L.A.; Graham, D.G. Intermediates in the Paal-Knorr synthesis of pyrroles. J. Org. Chem. 1991, 56, 6924-6931. [CrossRef]

46. OpenWetWare Contributors, Todd: Synthesis of Analogs of Arylpyrrole Antimalarial Drug Leads. Available online: http://www.openwetware.org/index.php?title=Todd:Synthesisof_Analogs_of_ Arylpyrrole_Antimalarial_Drug_Leads\&oldid=567645 (accessed on 20 February 2020).

47. Petrov, V.; Stanimirov, S.; Petrov, I.K.; Fernandes, A.; de freitas, V.; Pina, F. Emptying the $\beta$-Cyclodextrin Cavity by Light: Photochemical Removal of the trans-Chalcone of 4',7-Dihydroxyflavylium. J. Phys. Chem. A 2013, 117, 10692-10701. [CrossRef]

48. Vazquez-Vuelvas, O.F.; Enriquez-Figueroa, R.A.; Garcia-Ortega, H.; Flores-Alamo, M.; Pineda-Contreras, A. Crystal structure of the chalcone(E)-3-(furan-2-yl)-1-phenylprop-2-en-1-one. Acta Cryst. 2015, E71, 161-164. [CrossRef]

49. Kuhn, B.; Mohr, P.; Stahl, M. Intramolecular hydrogen bonding in medicinal chemistry. J. Med. Chem. 2010, 53, 2601-2611. [CrossRef]

50. Attram, H.D.; Wittlin, S.; Chibale, K. Incorporation of an intramolecular hydrogen bonding motif in the side chain of antimalarial benzimidazoles. Med. Chem. Commun. 2019, 10, 450-455. [CrossRef]

51. Elemental Composition Calculator. Available online: https://webapp.scs.illlinois.edu/microanalysis/calc/ (accessed on 16 December 2019).

52. Sheldrick, G.M. Crystal structure refinement with SHELXL. Acta Crystallogr. C 2015, 71, 3-8. [CrossRef]

53. Barbour, L.J. X-Seed-A software tool for supramolecular crystallography. J. Supram. Chem. 2001, 1, $189-191$. [CrossRef]

54. Hirymi, H.; Hirumi, K. Continous cultivation of Trypanosoma brucei blood stream forms in a medium containing a low concentration of serum protein without feeder cell layers. J. Parasitol. 1989, 75, 985-989. [CrossRef] 
55. Oderinlo, O.O.; Tukulula, M.; Isaacs, M.; Hoppe, H.C.; Taylor, D.; Smith, V.J.; Khanye, S.D. New thiazolidine-2,4-dione derivatives combined with organometallic ferrocene: Synthesis, structure and antiparasitic activity. Appl. Organomet. Chem. 2018, 32, e4385. [CrossRef]

56. Beteck, R.M.; Legoabe, L.J.; Isaacs, M.; Khanye, S.D.; Laming, D.; Hoppe, H.C. Anti-trypanosomal and antimalarial properties of tetralone derivatives and structurally related benzocycloalkanones. Medicina 2019, 55, 206. [CrossRef] [PubMed]

Sample Availability: Samples of the compounds are available from the authors.

(C) 2020 by the authors. Licensee MDPI, Basel, Switzerland. This article is an open access article distributed under the terms and conditions of the Creative Commons Attribution (CC BY) license (http://creativecommons.org/licenses/by/4.0/). 